\title{
Magnetic structures in a dynamo simulation
}

\author{
By A. BRANDENBURG ${ }^{1}$, R.L. JENNINGS ${ }^{2}+$, \\ A. NORDLUND ${ }^{3,4}$, M. RIEUTORD ${ }^{5,6}$, R.F. STEIN ${ }^{7}$ \\ AND I. TUOMINEN 8.9
}

${ }^{1}$ Nordita, Blegdamsvej 17, DK-2100 Copenhagen $\varnothing$, Denmark

${ }^{2}$ DAMTP, University of Cambridge, Silver St, Cambridge CB3 9EW, UK

${ }^{3}$ Theoretical Astrophysics Center, Blegdamsvej 17, DK-2100 Copenhagen Ø, Denmark

${ }^{4}$ Copenhagen University Observatory, Øster Voldgade 3, DK-1350 Copenhagen, Denmark

${ }^{5}$ Observatoire Midi-Pyrénées, 14 av. E. Belin, F-31400 Toulouse, France

${ }^{\circ}$ CERFACS, 42, Avenue Coriolis, F-31057 Toulouse, France

${ }^{7}$ Dept. of Physics and Astronomy, Michigan State University, East Lansing, MI 48824, USA

${ }^{8}$ Observatory, PO Box 14, SF-00014 University of Helsinki, Finland

${ }^{9}$ Dept. of Geosciences and Astronomy, University of Oulu, PO Box 333, 90571 Oulu, Finland

(Received 14 May 1993 and in revised form 21 September 1995)

We use three-dimensional simulations to study compressible convection in a rotating frame with magnetic fields and overshoot into surrounding stable layers. The, initially weak, magnetic field is amplified and maintained by dynamo action and becomes organized into flux tubes that are wrapped around vortex tubes. We also observe vortex buoyancy which causes upward flows in the cores of extended downdraughts. An analysis of the angles between various vector fields shows that there is a tendency for the magnetic field to be parallel or antiparallel to the vorticity vector, especially when the magnetic field is strong. The magnetic energy spectrum has a short inertial range with a slope compatible with $k^{+1 / 3}$ during the early growth phase of the dynamo. During the saturated state the slope is compatible with $k^{-1}$. A simple analysis based on various characteristic timescales and energy transfer rates highlights important qualitative ideas regarding the energy budget of hydromagnetic dynamos.

\section{Introduction}

The dynamo action of magnetohydrodynamic turbulence is of major importance in virtually all astrophysical bodies. In stars, accretion discs and galaxies the turbulence is compressible and affected by rotation and stratification. Here we focus on solar-type stars, where low Prandtl number turbulence is driven by convection which penetrates into the lower radiative interior.

Previous investigations of convective dynamos have concentrated on the magnetic field evolution either on large scales using spherical geometry (e.g. Gilman 1983; Glatzmaier 1985; Valdettaro \& Meneguzzi 1991), or on small scales using Cartesian geometry (Meneguzzi \& Pouquet 1989). In a recent paper (Nordlund et al. 1992, hereafter referred to as Paper I) we included, for the first time, the combined effects of compressibility, rotation, stratification, overshoot, and low Prandtl

† Present address: Shell Research BV, Postbus 60, 2280 AB Rijswijk, The Netherlands. 

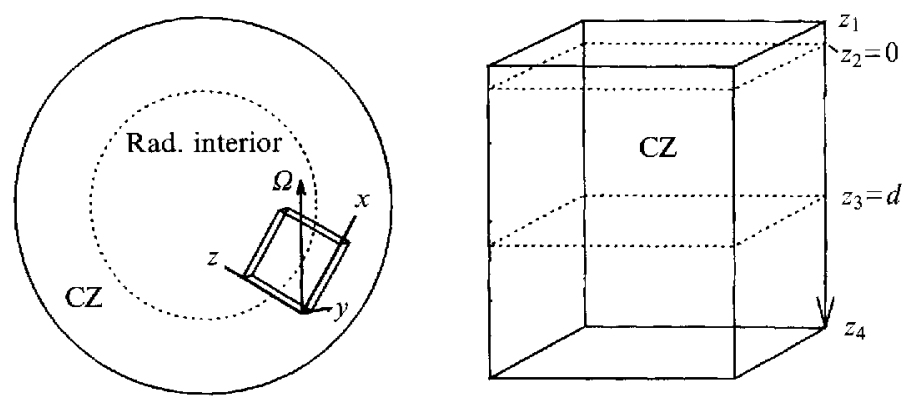

FIGURE 1. A Cartesian box in the convection zone at a southern latitude, with $z$ parallel to $g$ and the rotation axis in the $(x, z)$-plane. The structure of the box and the interfaces at $z_{1} \ldots z_{4}$ are shown on the right-hand side. $\mathrm{CZ}$ denotes convection zone.

number in a Cartesian geometry. These simulations provide new insights into convective magnetohydrodynamic turbulence, which laboratory experiments could not provide.

The goal of this paper is to present new results obtained in such high-resolution numerical experiments and to investigate the magnetic structures associated with the dynamo process and their correlation with other fluid properties. The magnetic structures in many respects resemble the vorticity tubes found in hydrodynamical turbulence, of which a detailed investigation has recently been carried out by Vincent \& Meneguzzi (1991, 1994). A corresponding investigation for the magnetic counterpart does not exist. Our simulations therefore provide a unique opportunity to carry out such studies and to test ideas such as the similarity between the dynamics of vorticity and magnetic fields, as proposed by Batchelor (1950).

The paper is organized as follows. After describing the simulation characteristics (e.g. equations of the flow, initial and boundary conditions, numerical method, etc.) we present some results about swirling convective downdraughts $(\$ 3)$ which drive the dynamo that amplifies a seed magnetic field. Three different aspects of the simulation data are then presented: the magnetic field structures (\$4), the statistics $(\S 5)$ and the spectra (\$6) of different physical quantities. A final discussion concludes the paper.

\section{The simulation}

\subsection{Equations}

We employ Cartesian coordinates, where the $x$-direction points along the meridian from south to north, the $y$-direction points in the azimuthal direction of rotation, and $z$ points downward in the direction of gravity $\boldsymbol{g}$, with $\boldsymbol{g}=g \hat{z}$ (figure 1 ). The rotation vector $\boldsymbol{\Omega}$ is inclined to $\boldsymbol{g}$ at an angle $\theta$, i.e. $\hat{\boldsymbol{g}} \cdot \hat{\boldsymbol{\Omega}}=\cos \theta$. Throughout this work we take $\theta=\pi / 3$ which means that the latitude of the domain is $30^{\circ}$ south of the equator. The computational domain (figure 1) has an unstable layer of depth $d$ between heights $z_{2}<z<z_{3}$, with stable overshoot layers $z_{1}<z<z_{2}$ above the convective layer and $z_{3}<z<z_{4}$ below the convective layer (as in Hurlburt, Toomre $\&$ Massaguer 1986). The horizontal extent is $2 d$ in each direction (except in Run B, where it is $3 d$ ). 
We solve the equations for conservation of mass, momentum, energy, and the induction equation for constant magnetic diffusivity $\eta$ :

$$
\begin{gathered}
\frac{\mathrm{D} \ln \rho}{\mathrm{D} t}=-\nabla \cdot \boldsymbol{u}, \\
\frac{\mathrm{D} \boldsymbol{u}}{\mathrm{D} t}=-(\gamma-1)(e \nabla \ln \rho+\nabla e)+\boldsymbol{g}-2 \boldsymbol{\Omega} \times \boldsymbol{u}+\frac{1}{\rho} \boldsymbol{J} \times \boldsymbol{B}+\frac{1}{\rho} \nabla \cdot \tau, \\
\frac{\mathrm{D} e}{\mathrm{D} t}=-(\gamma-1) e \nabla \cdot \boldsymbol{u}+\frac{1}{\rho} \nabla(\mathscr{K} \nabla e)+2 v \boldsymbol{s}^{2}+\frac{\eta \mu_{0}}{\rho} \boldsymbol{J}^{2}+Q, \\
\frac{\partial \boldsymbol{B}}{\partial t}=\nabla \times(\boldsymbol{u} \times \boldsymbol{B})+\eta \nabla^{2} \boldsymbol{B},
\end{gathered}
$$

respectively, where $\mathrm{D} / \mathrm{D} t=\partial / \partial t+\boldsymbol{u} \cdot \nabla$ is the total derivative, $\mathscr{K}$ the radiative conductivity divided by $c_{v}, \boldsymbol{J}=\boldsymbol{\nabla} \times \boldsymbol{B} / \mu_{0}$ the electric current, $\mu_{0}$ the vacuum permeability, and the stress tensor $\tau=2 v \rho S$, where

$$
S_{i j}=\frac{1}{2}\left(\partial_{j} u_{i}+\partial_{i} u_{j}-\frac{2}{3} \delta_{i j} \partial_{k} u_{k}\right) .
$$

We take the kinematic viscosity $v$ as constant throughout the convection zone. A constant $\rho v$ with a strong density stratification would have made the upper layers significantly more dissipative than the lower layers, which is unrealistic because the dissipation should be as small as possible for a given resolution. (For a monatomic gas such as ionized hydrogen or helium the bulk viscosity is zero.)

We assume a perfect gas law

$$
p=(\gamma-1) \rho e,
$$

with constant specific heats, $c_{p}$ and $c_{v}$, and with $\gamma=c_{p} / c_{v}=5 / 3$. In all the runs where $z_{1}<0$, we adopt a cooling function $Q$ of the form

$$
Q=-\sigma_{0} f(z)\left(e-e_{\text {top }}\right),
$$

where $\sigma_{0}$ is the cooling rate and $f(z)$ is a profile function that vanishes everywhere except close to the surface $\left(z_{1} \leqslant z \leqslant z_{2}\right)$ where $f(z)=1$.

\subsection{Initial and boundary conditions}

On the upper and lower boundaries of our computational domain we impose conditions which are mathematically convenient, yet physically plausible. By including overshoot layers the hope is that the actual conditions imposed at the top and bottom have little influence on the flow in the convection zone (CZ).

At the top we impose a constant temperature and at the bottom a constant energy flux; that is

$$
\begin{aligned}
e & =e_{\text {top }} \quad \text { at } \quad z=z_{1}, \\
\mathrm{de} / \mathrm{d} z & =(\mathrm{d} e / \mathrm{d} z)_{\text {bot }} \quad \text { at } \quad z=z_{4} .
\end{aligned}
$$

We assume the upper and lower boundaries to be impenetrable stress-free perfect conductors which implies

$$
\left.\begin{array}{r}
\partial_{z} u_{x}=\partial_{z} u_{y}=u_{z}=0 \\
\partial_{z} B_{x}=\hat{o}_{z} B_{y}=B_{z}=0
\end{array}\right\} \quad \text { at } z=z_{1}, z_{4} .
$$

In addition we require all quantities to be periodic in the two horizontal directions. With the above boundary conditions, a manipulation of the induction and continuity 
equations reveals that each component of the magnetic flux vector and the total mass in the domain are conserved.

In the initial state we set $\boldsymbol{u}=\boldsymbol{B}=\partial / \partial t=0$ in (2.4)-(2.1) and obtain equations for the hydrostatic and radiative equilibrium which depend upon $m(z)$ :

$$
\begin{aligned}
& \mathrm{d} e / \mathrm{d} z=m_{a d} g /[m(z)+1], \\
& \mathrm{d} \ln \rho / \mathrm{d} z=m(z) \mathrm{d} \ln e / \mathrm{d} z .
\end{aligned}
$$

We specify the normalized pressure scale height $\xi_{0}$ at the top of the domain, where $\xi_{0}=H_{p}^{(t o p)} / d, H_{p}^{(t o p)}=(\gamma-1) e_{\text {top }} / g$, and $e_{\text {top }}$ is expressed in terms of $\xi_{0}$ by

$$
e_{t a p}=\xi_{0} m_{a d} g d,
$$

where $m_{a d}=1 /(\gamma-1)=3 / 2$ is the polytropic index for an adiabatic stratification. Small values of $\xi_{0}$ correspond to strong stratification. The actual stratification after a statistically stationary state is obtained is measured by the number of scale heights over either the full box, $\Delta \ln \langle p\rangle$, or within the unstable layer, $\Delta \ln \langle p\rangle_{C Z}$.

Convective instability occurs in the interior of a star when $\mathscr{K}(\rho, e)$ is smaller than a critical value. The dependence $\mathscr{K}=\mathscr{K}(\rho, e)$ in stars is highly nonlinear with large variations over many orders of magnitude. Here, for simplicity we prescribe a vertical profile $\mathscr{K}(z)$ such that the middle layer is convectively unstable and the other two are stable to convection (Hurlburt et al. 1986). In each layer $\mathscr{K}$ is a different constant, $\mathscr{K}_{i}(i=1,2,3)$ and these different values are joined smoothly in thin transition layers of thickness $0.1 d$ using third-order polynomials, so that $\mathscr{K}(z)$ is continuous and differentiable. The value of $\mathscr{K}_{2}$ in the unstable layer is determined by the values we prescribe for the Rayleigh and Prandtl numbers (see $\$ 2.3$ ). Since $\mathscr{K}$ is a function of $z$ so is the polytropic index $m$. In stellar convection theory $\nabla_{\text {rad }}=1 /(m+1)$ is sometimes used instead of $m$. A relation between $m$ and $\mathscr{K}$ in each layer is obtained by requiring the total flux of the initial state, $\mathscr{K}_{i} \mathrm{~d} e / \mathrm{d} z=F_{t o i}$, to remain constant. Using (2.11)

$$
m_{i}+1=\mathscr{K}_{i} m_{a d} g / F_{\text {tot }}, \quad i=1,2,3 .
$$

In all cases studied below we adopted $m_{1}=m_{2}=1$ and $m_{3}=3$, so the system is convectively unstable in the upper and middle parts of the box and stably stratified in the lower part. In those cases where $z_{1}<0$ we included cooling with $\sigma=10$, which tends to make the upper part isothermal and thus convectively stable.

This equilibrium is perturbed by the addition of a small-amplitude random velocity field. When the convection has reached a statistically stationary state a seed magnetic field is added to the convectively unstable region. In most cases we used $\boldsymbol{B}=\left(B_{x}, 0,0\right)$ where

$$
\begin{aligned}
B_{x} & =B_{\text {seed }} \sin \left[2 \pi\left(z-z_{a}\right) /\left(z_{b}-z_{a}\right)\right] \text { for } z_{a}<z<z_{b} \\
& =0 \text { elsewhere }
\end{aligned}
$$

with $z_{a}=0.3 d$ and $z_{b}=0.7 d$. With this seed field $\int \boldsymbol{B} \mathrm{d}^{3} x=\mathbf{0}$ for all time. In some cases a random initial magnetic field was used.

\subsection{Dimensionless quantities}

After setting

$$
d=g=\bar{\rho}=\mu_{0}=c_{p}=1,
$$

we measure length in units of $d$, time in units of $(d / g)^{1 / 2}$ (which is $\sqrt{2}$ times the freefall time of the unstable layer), density in units of $\bar{\rho} \equiv \int \rho \mathrm{d}^{3} x / \int \mathrm{d}^{3} x$, and magnetic 
field in units of $\left(\mu_{0} \bar{\rho} g d\right)^{1 / 2}$. We define the Rayleigh number as

$$
R a=\frac{g d^{4}}{v \bar{\chi} c_{p}}\left(\frac{\mathrm{d} s}{\mathrm{~d} z}\right)_{0}
$$

where $\bar{\chi}=\mathscr{K}_{2} / \gamma \bar{\rho}$ is an average radiative diffusivity, and $(\mathrm{d} s / \mathrm{d} z)_{0}$ is the entropy gradient in the middle of the initial stratification. It can be shown that

$$
\frac{d}{c_{p}}\left(\frac{\mathrm{d} s}{\mathrm{~d} z}\right)_{0}=2 \frac{m_{a d}-m_{2}}{m_{a d}+1} \frac{1}{1+2 \xi_{0}\left(m_{2}+1\right)}
$$

where

$$
\frac{m_{a d}-m_{2}}{m_{a d}+1}=\frac{\mathscr{K} g}{\gamma F_{\text {tot }}}-1=1-\frac{\nabla_{a d}}{\nabla_{r a d}} .
$$

Rotation is measured by the Taylor number $T a$, which is chosen so that the inverse Rossby number, $R o^{-1}=2 \Omega d / u_{r m s}$, is of order unity, as in the Sun. Two Prandt1 numbers also need to be defined:

$$
\operatorname{Pr}=v / \bar{\chi}, \quad \operatorname{Pr}_{M}=v / \eta .
$$

The whole system is described by 11 dimensionless numbers: $\gamma, \operatorname{Pr}, \operatorname{Pr}_{M}, \operatorname{Ra}, T a, \theta$, $\xi_{0}, \sigma_{0}, m_{1}, m_{2}, m_{3}$, plus those which describe the domain's geometry.

\subsection{Characteristic time and length scales}

Important timescales are the diffusion and dissipation times. The $e$-folding time for the decay of the magnetic field in the absence of induction effects is the ratio between the magnetic energy and the Joule dissipation, $\tau_{M}=E_{M} / Q_{J}$, which is also the magnetic diffusion timescale based on the magnetic Taylor microscale

$$
\lambda_{M}=\left(5\left\langle\boldsymbol{B}^{2}\right\rangle /\left\langle\mu_{0}^{2} \boldsymbol{J}^{2}\right\rangle\right)^{1 / 2},
$$

that is $\tau_{M}=\left(1 / 2 \mu_{0}\right)\left\langle\boldsymbol{B}^{2}\right\rangle /\left\langle\eta \mu_{0} J^{2}\right\rangle=\frac{1}{10} \lambda_{M}^{2} / \eta$, which is typically much shorter than the global magnetic diffusion timescale $d^{2} / \eta$. (The factor 5 in (2.21) is included to give agreement with the standard definition of the Taylor microscale in one dimension.) Timescales for the decay of kinetic energy and vorticity are $\tau_{K}=\frac{1}{10} \lambda_{K}^{2} / v$, and $\tau_{\omega}=\frac{1}{10} \lambda_{\omega}^{2} / v$, where

$$
\lambda_{K}=\left(5\left\langle u^{2}\right\rangle /\left\langle\omega^{2}\right\rangle\right)^{1 / 2}, \quad \lambda_{\omega}=\left(5\left\langle\omega^{2}\right\rangle /\left\langle|\nabla \times \omega|^{2}\right\rangle\right)^{1 / 2},
$$

the kinetic Taylor microscale and vorticity microscale, respectively (Pouquet \& Patterson 1978; Lesieur 1990). The total viscous dissipation is actually $\epsilon=v\left[\left\langle\omega^{2}\right\rangle+\right.$ $\left.\frac{2}{3}\left\langle(\operatorname{div} \boldsymbol{u})^{2}\right\rangle\right]$, but in the runs considered in this paper $\left\langle(\operatorname{div} u)^{2}\right\rangle \lesssim 0.01\left\langle\boldsymbol{\omega}^{2}\right\rangle$.

\subsection{The numerical method}

Equations (2.1)-(2.4) together with the appropriate boundary conditions are solved numerically using a modified version of the code by Nordlund \& Stein (1990). Advances in time are achieved either by a second-order Adams-Bashforth scheme (Run A) or by a third-order Hyman (1979) scheme (all other runs). Spatial derivatives are calculated from cubic splines. Timesteps are usually $25 \%$ of the CourantFriedrich-Levy timestep, which in our model is defined as $\delta x / u_{C F L}$, with $\delta x$ a single mesh interval and $u_{C F L}=\max \left(c, v_{A},|\boldsymbol{u}|, q v / \delta x, q \bar{\chi} / \delta x\right)$, where $c$ is the sound speed, $v_{A}$ the Alfvén speed, and $q$ is an empirical factor (around 0.1). It is usually the sound speed which limits the timestep, and only in runs with high resolution and small values of $R a$ and $P r$ does the diffusive restriction become important (Run O). A number of 


$\begin{array}{cccccc}\text { Run } & \mathrm{O} & \mathrm{A} & \mathrm{B} & \mathrm{C} & \mathrm{D} \\ \text { Mesh } & 126^{2} \times 105 & 63^{2} \times 63 & 95^{2} \times 63 & 126^{2} \times 105 & 126^{2} \times 105 \\ z_{1} & -0.15 & 0.0 & -0.15 & -0.15 & -0.15 \\ z_{4} & 1.5 & 2.0 & 1.85 & 1.5 & 1.5 \\ \xi_{0} & 0.1 & 0.2 & 0.2 & 0.1 & 0.1 \\ \sigma_{0} & 10 & 0 & 10 & 10 & 10 \\ R a & 10^{6} & 10^{6} & 3 \times 10^{6} & 3 \times 10^{7} & 3 \times 10^{7} \\ P r & 0.1 & 0.2 & 0.2 & 0.1 & 0.1 \\ \operatorname{Pr}_{M} & 1.0 & 4.0 & 0.5 & 1.0 & 0.5 \\ \operatorname{Re} & 140 & 310 & 540 & 1100 & 1200 \\ \operatorname{Re}_{M} & 140 & 1240 & 270 & 1100 & 600 \\ \operatorname{Re}_{i} & 35 & 63 & 89 & 121 & 123 \\ R 0^{-1} & 7.1 & 3.2 & 3.2 & 5.0 & 4.6 \\ \lambda_{K} & 0.25 & 0.20 & 0.17 & 0.10 & 0.11 \\ \lambda_{M} & 0.06 & 0.08 & 0.07 & 0.03 & 0.03 \\ \tau_{M} & 1.8 & 10.9 & 2.5 & 6.1 & 2.7 \\ \Delta \ln \langle p\rangle & 6.0 & 3.9 & 4.6 & 6.4 & 6.4 \\ \Delta \ln \langle p\rangle_{C Z} & 3.8 & 2.6 & 2.6 & 4.1 & 4.1\end{array}$

TABLE 1. Summary of data from numerical simulations. The averages are taken over the whole box.

runs are summarized in table 1 , of which only Runs $\mathrm{A}$ and $\mathrm{D}$ are investigated below. The other runs are included to facilitate comparison of various Reynolds numbers, time and length scales, and vertical stratification. In Run $O$ the magnetic field is decaying and in Run B a horizontal magnetic field is applied, i.e. these runs are not dynamos. Runs $\mathrm{A}, \mathrm{C}$, and $\mathrm{D}$ show dynamo action and the quantities in table 1 refer to a state where the dynamo is saturated.

\section{Swirling downdraughts}

Video animations suggest that the magnetic field evolution is primarily controlled by swirling downdraughts. We therefore begin by inspecting these downdraughts in more detail. The turbulent motions are conveniently visualized using images of the magnitude of the vorticity, $|\omega|$, and of the normalized helicity density $H=$ $\boldsymbol{\omega} \cdot \boldsymbol{u} /\left(\omega_{r m s} u_{r m s}\right)$, where $\omega_{r m s}$ and $\boldsymbol{u}_{r m s}$ are the root-mean-square values of the vorticity and velocity, respectively. The vertical cross-section (figure 2) reveals the complex nature of the downdraughts which appear to be reminiscent of the turbulent plumes that are believed to exist in the solar CZ (Rieutord \& Zahn 1995). Figure 2(b) shows that the helicity is large and of either sign within such descending turbulent plumes.

These downdraughts are important for the dynamo, because they are able to wind up magnetic flux tubes into a spiral, which could lead to an amplification of magnetic flux analogous to the shear-twist-fold dynamo of Vainshtein \& Zeldovich (1972). The degree of twisting is measured by the helicity. However, figure 2 shows that the helicity associated with the downdraughts can have either sign. Taken over the whole box the relative helicity is small $(-0.03)$ due to cancellations.

The dynamics of the downdraughts is made significantly more complicated by anomalous density reversals in their cores. Fluid in the centre of a downdraught is usually heavier than its surroundings. However, under the influence of the Coriolis force, a downdraught generates vorticity which leads to an evacuation of mass at the very core of the vortex, as in a cyclone (figure $3 a$ ). The buoyancy associated with this 
light fluid decelerates the downward flow and can even cause it to reverse (figure $3 b$ ). We refer to this effect as vortex buoyancy. (Comparison with a snapshot taken at an earlier time with a weaker magnetic field shows similar behaviour, indicating that the magnetic field has little influence on this effect.)

We explain this reduction in density as follows: rotation imparts vorticity to the downdraught resulting in large local horizontal velocities. Horizontal force balance requires a central pressure reduction of the order of the dynamical pressure in the horizontal motions. The lengthscale on which temperature varies is much larger than the scale of the downdraught because the Prandtl number is small. Thus according to $\rho \propto p / e$, the density reduction is primarily due to the reduction in pressure in the downdraughts. For a larger value of the Prandtl number (not shown) we did not find such a density reversal, indicating that this is indeed a combined effect of low Prandtl number and rapid rotation. It is mainly the dynamical pressure due to the horizontal motions which leads to the evacuation.

The effect of vortex buoyancy reverses the 'buoyancy braking' mechanism. In compressible convection without rotation there are positive pressure fluctuations above both upwellings and downdraughts. This gives positive density fluctuations which brake the upwellings and accelerate the downdraughts, and hence enhance the well known up/down asymmetry in compressible convection of high-speed descent in thin downdraughts and a low-speed rise in broad upwellings (Toomre et al. 1976; Hurlburt, Toomre \& Massaguer 1984). Here though, with rotation, this asymmetry is less pronounced because there is less enhancement of the density fluctuation due to the reduction of pressure in downdraughts via the mechanism described above. A similar connection between low-pressure regions and vortex tubes has recently been discussed by Douady, Couder \& Brachet (1991).

An investigation of the formation of those buoyant vortices is beyond the scope of the present paper and will be addressed in a separate paper.

\section{The dynamo}

For large values of $R e_{M}$ the non-magnetic state is unstable and magnetic field perturbations grow until saturated by nonlinear effects. In Paper I we described the amplification of such a seed field in the kinematic regime where the Lorentz force is negligible as well as in the dynamic regime where the magnetic field affects the flow. Apart from a brief resume of the dynamo process we concentrate here upon the magnetic field structure, and the statistics of the hydromagnetic flow.

\subsection{Amplification of a seed magnetic field}

In figure 4 we show the growth of magnetic energy versus time for two runs with different initial seed field energies in which $P r=0.2$ and $P r_{M}=4$. The seed field was either a solenoidal random field, curve (i), or $B_{x}$ was computed from (2.15), curve (ii). In these runs the magnetic energy increases by 3 and 5 orders of magnitude, respectively.

During the growth phase of the dynamo the magnetic Taylor microscale increases continuously and levels off at approximately 0.07 as the dynamo saturates. This increase is associated with the magnetic flux tubes becoming stronger and more robust as the dynamo settles. We also found that $\lambda_{M}$ is fairly constant throughout the convection zone, but becomes larger both close to the surface and in the lower overshoot layer where convective motions are rather weak. Larger magnetic diffusivity (smaller values of $P r_{M}$ ) yields only slightly larger values of $\lambda_{M}$. 

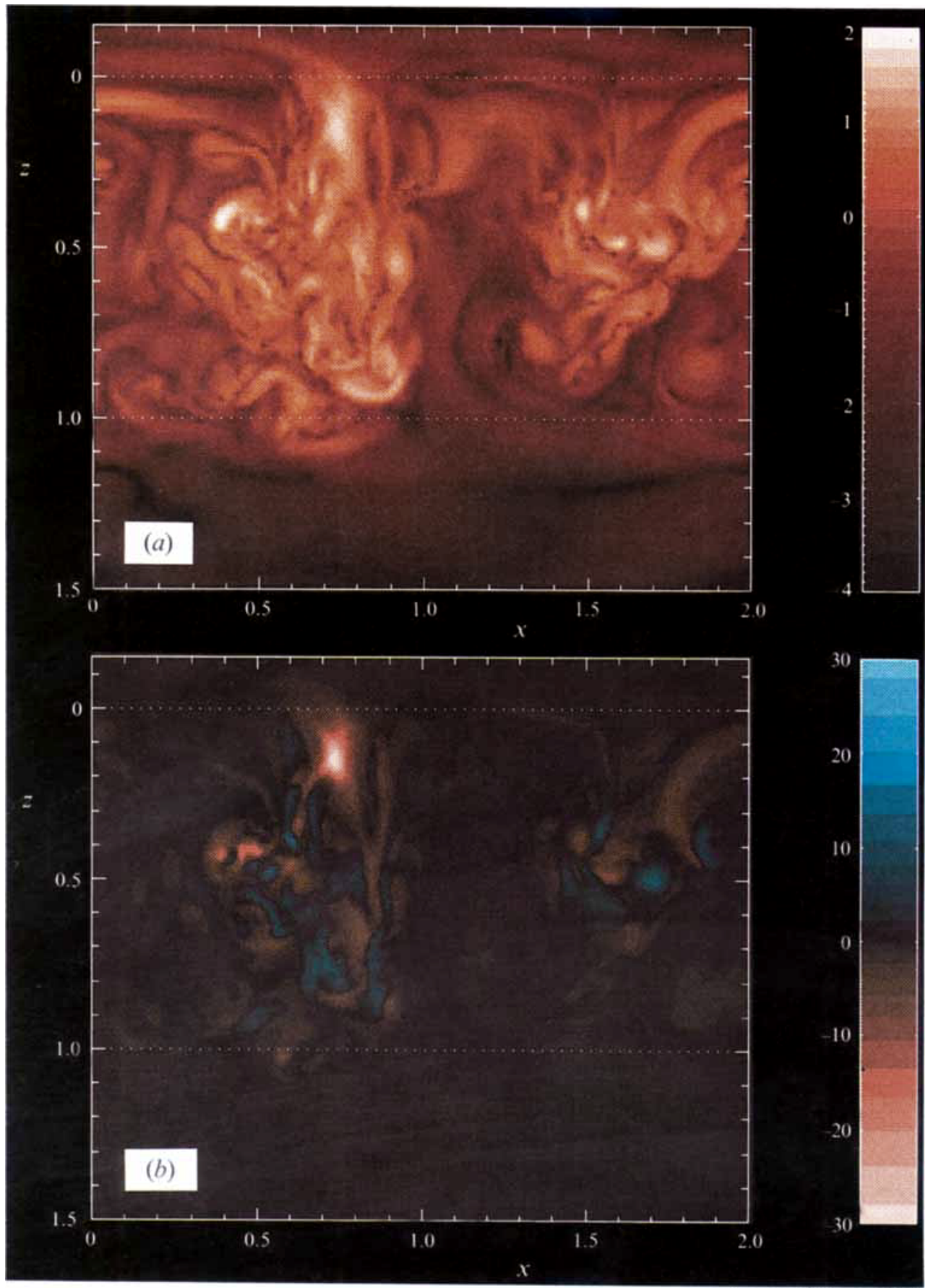

FIGURE 2. (a) The logarithm of the magnitude of the vorticity in a vertical cross-section $(y=1.88)$. Note the turbulent structure of the downdraughts compared to the more laminar upwellings. (b) The normalized helicity density $I$ in the same plane as above. Note that large helicities occur in the downdraughts. Run D. 


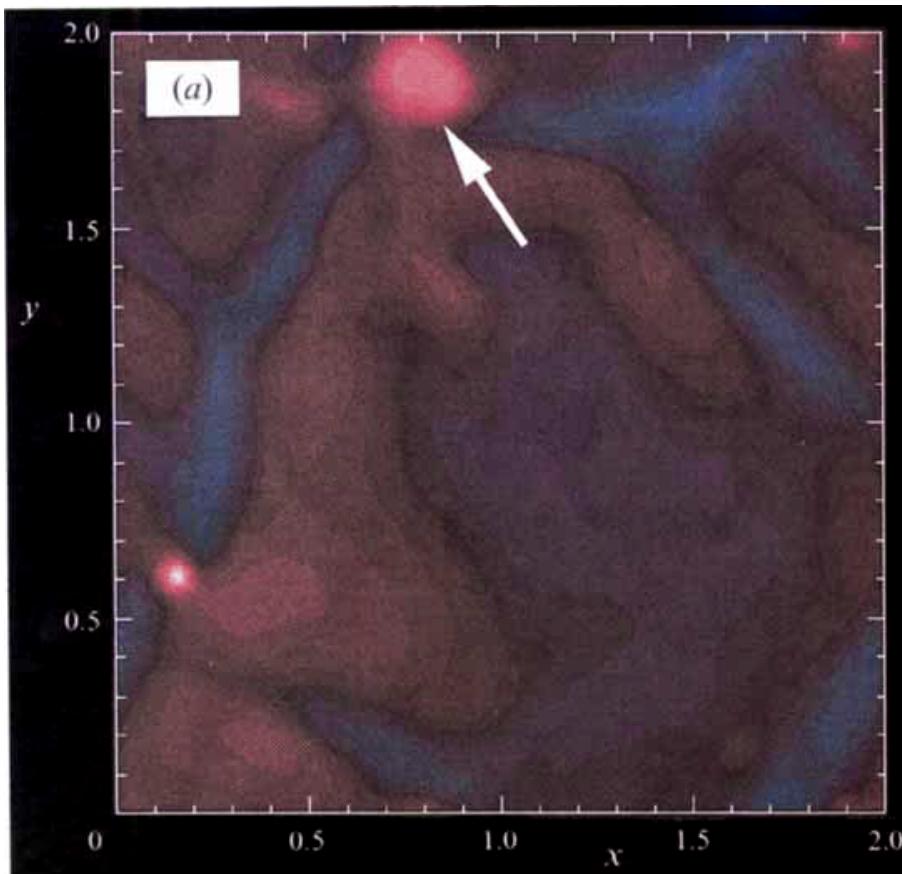

0.04
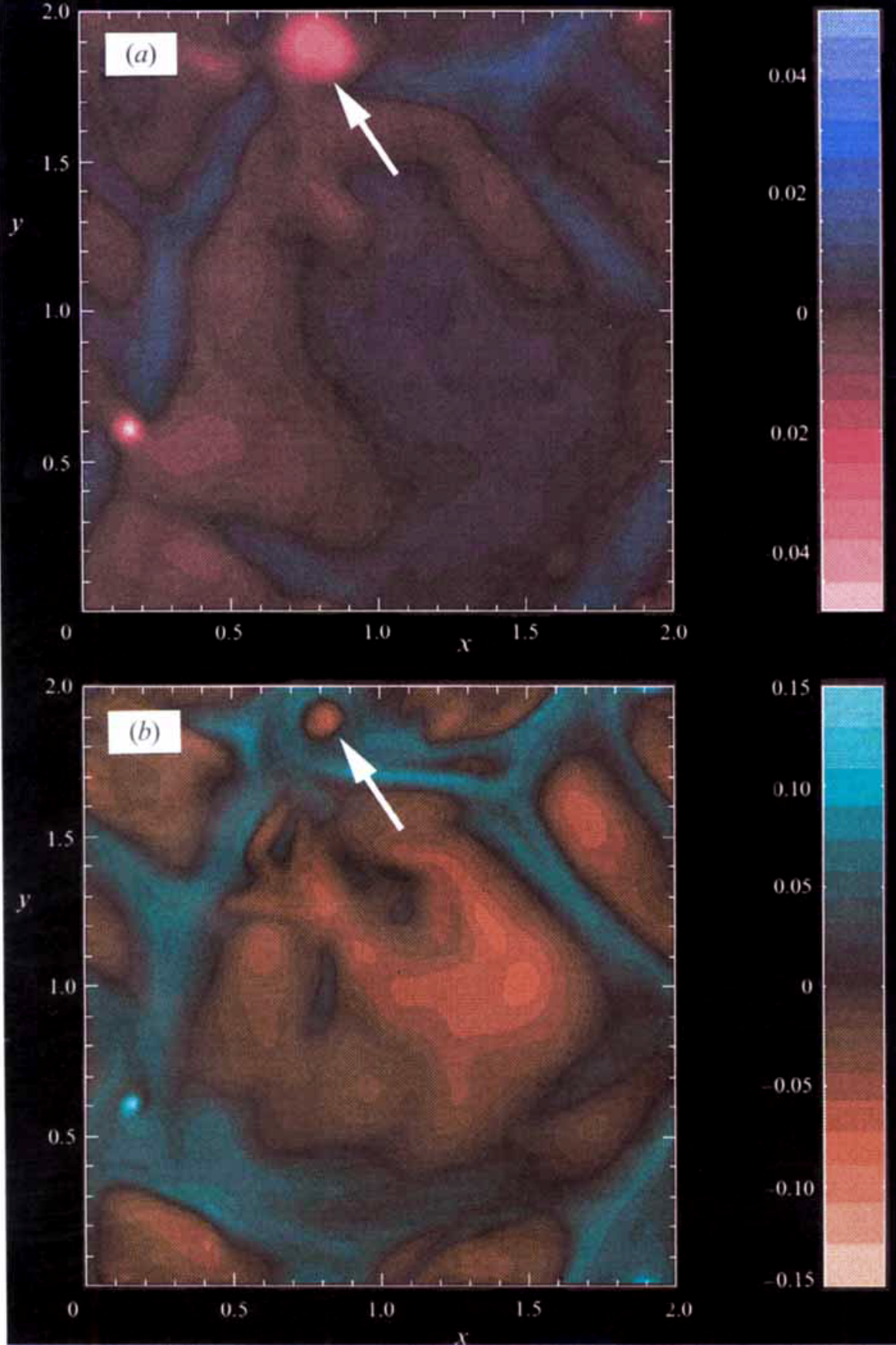

Figure 3. (a) Horizontal image of the relative density fluctuation $\rho^{\prime} / \rho$. Light, low-density regions are pink. (b) Vertical velocity normalized by the sound speed, $u_{z} / c$. The arrows indicate a strong swirling downdraught where vortex buoyancy leads to an upward motion in the core of the vortex. Run D. 
(a)

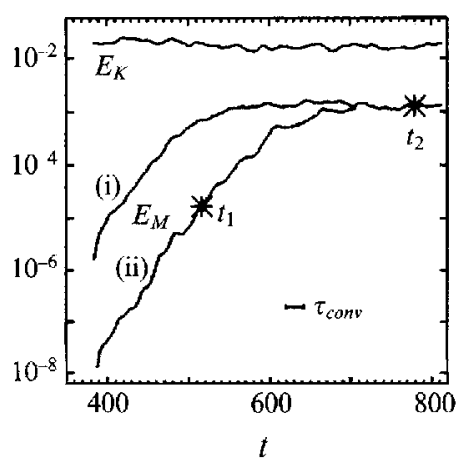

(b)

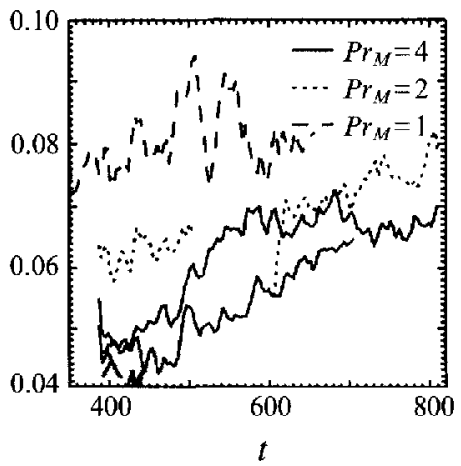

FIGURE 4. (a) Exponential growth of magnetic encrgy $E_{M}$ over 3 and 5 orders of magnitude and subsequent saturation. Note that the saturation amplitude is independent of the seed field's strength and that the growth is on the convective turnover timescale $\tau_{c o n v}$. As time increases there is a slight decrease in the total kinetic energy $E_{K}$. Run A. (b) Time series of the magnetic Taylor microscale $\lambda_{M}$. The solid line refers to Run A, and the dotted and dashed lines to similar runs, but with different values of $\operatorname{Pr}_{M}$.

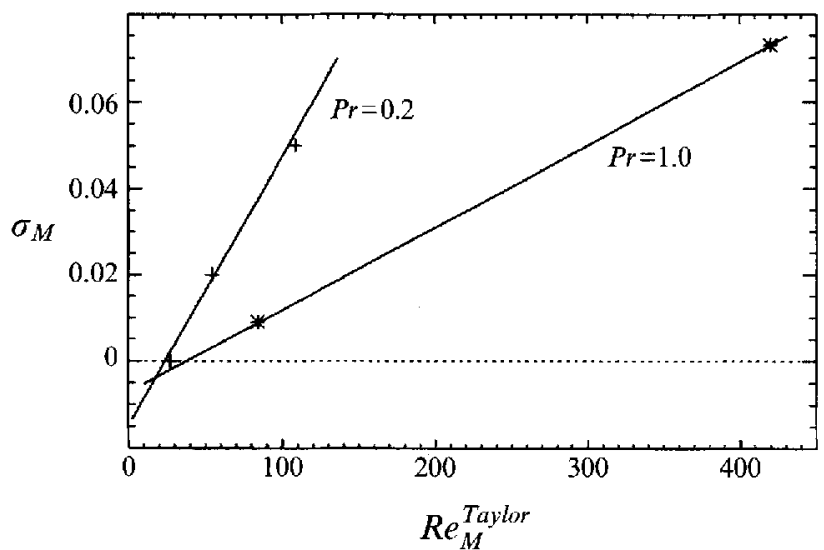

FIGURE 5. Growth rate $\sigma_{M}$ of the dynamo for different values of the magnetic Reynolds number based on the Taylor microscale.

The values of $\lambda_{M}$ (cf. table 1) are just a few times the mesh size and one might be worried that the numerical resolution is therefore insufficient. However, magnetic flux tubes are typically $6 \lambda_{M}$ wide. In $\S 6.3$ we compute the spatial autocorrelation function and find that the lengthscale at which this function has the first transition through zero is approximately 0.1 (or about 6 mesh sizes for Run D), which we consider as evidence of sufficient resolution.

Cattaneo, Hughes \& Weiss (1991) raised the question of how long one has to run a simulation before one can claim to have a dynamo. They proposed a minimum time $\tau_{M} f_{\text {safety }}$, where $f_{\text {safety }}=(L / l)^{2}$ is a safety factor, which is the square of the ratio of the scale of the box to a small scale. Assuming $l=\lambda_{K}$ (see $\S \S 2.4$ and 2.5), the minimum time would be around $10 \times(1 / 0.2)^{2}=250$. Note that the integration time in figure 4 exceeds this value.

We have computed the growth rate $\sigma_{M}=\frac{1}{2}(\mathrm{~d} / \mathrm{d} t) \ln E_{M}$ of the dynamo for different values of $\mathrm{Pr}$. In figure 5 we plot $\sigma_{M}$ for different values of the magnetic Reynolds number based on the Taylor microscale, that is, $R e_{M}^{\text {Taylor }}=u_{t} \lambda_{K} / \eta$. For the run with $\operatorname{Pr}=1$ we have only two values. Nevertheless, the graph suggests that the critical 


$$
\begin{aligned}
& E_{M}=\frac{1}{2 \mu_{0}} \int \boldsymbol{B}^{2} \mathrm{~d} \boldsymbol{V} \quad \boldsymbol{W}_{L}=\int \boldsymbol{u} \cdot(\boldsymbol{J} \times \boldsymbol{B}) \mathrm{d} V \quad Q_{3}=\eta \mu_{0} \int \boldsymbol{J}^{2} \mathrm{~d} \boldsymbol{V} \\
& E_{K}=\frac{1}{2} \int \rho \boldsymbol{u}^{2} \mathrm{~d} \boldsymbol{V} \quad \boldsymbol{W}_{c}=\int p \nabla \cdot \boldsymbol{u} \mathrm{d} V \quad Q_{v}=2 v \int \rho \boldsymbol{S}^{2} \mathrm{~d} \boldsymbol{V} \\
& E_{P}=-\int \rho \boldsymbol{g} \cdot \boldsymbol{z} \mathrm{d} \boldsymbol{V} \quad W_{b}=\int \rho \boldsymbol{g} \cdot \boldsymbol{u} \mathrm{d} V \\
& E_{T}=\int \rho e \mathrm{~d} V \quad L_{b o t}=\int(\mathscr{K} \mathrm{d} e / \mathrm{d} z)_{b o t} \mathrm{~d} x \mathrm{~d} y \quad L_{t o p}=\int(\mathscr{K} \mathrm{d} e / \mathrm{d} z)_{t o p} \mathrm{~d} x \mathrm{~d} y
\end{aligned}
$$

TABLE 2. Definition of the different terms in the energy equations (4.1)-(4.4).

value for dynamo action is approximately $30-40$, and seems to be independent of (or only weakly dependent on) $\mathrm{Pr}$. It is interesting to note that the onset of dynamo action in the model of Meneguzzi \& Pouquet (1989) is characterized by a similar value of the magnetic Reynolds number based on the Taylor microscale of around 30-40. The Reynolds number based on the integral scale, however, is rather different in their and our models. This result suggests that, while the onset of dynamo action can depend on a number of different parameters, the critical magnetic Reynolds number based on the Taylor microscale seems to be a useful quantity to quantify the onset under various circumstances.

\subsection{Energy budget}

Energy equations may be derived from the basic equations (2.1)-(2.4) and the boundary conditions (2.8) $-(2.10)$. We obtain a set of four equations:

$$
\begin{gathered}
\frac{\mathrm{d} E_{M}}{\mathrm{~d} t}=-W_{L}-Q_{J}, \\
\frac{\mathrm{d} E_{K}}{\mathrm{~d} t}=W_{c}+W_{b}+W_{L}-Q_{V}, \\
\frac{\mathrm{d} E_{P}}{\mathrm{~d} t}=-W_{b}, \\
\frac{\mathrm{d} E_{T}}{\mathrm{~d} t}=-W_{c}+L_{b o t}-L_{t o p}+Q_{V}+Q_{J},
\end{gathered}
$$

for the magnetic, $E_{M}$, kinetic, $E_{K}$, gravitational potential, $E_{P}$, and thermal, $E_{T}$, energy; $W_{c}, W_{b}, W_{L}$, are the work done by compression, buoyancy and by the Lorentz force; $Q_{J}, Q_{V}$ are Joule and viscous heating; and $L_{b o t}$ and $L_{t o p}$ are the luminosities at top and bottom. The total energy satisfies

$$
\frac{\mathrm{d}}{\mathrm{d} t}\left(E_{M}+E_{K}+E_{P}+E_{T}\right)=L_{b o t}-L_{t o p} .
$$

The definition of various terms is given in table 2 .

The work done by buoyancy describes the change of the density stratification. During the start-up phase when the system changes from a hydrostatic stratification to a convective state, $W_{b}$ is important. Even during the statistically steady convective state $W_{b}$ is in general non-vanishing due to the presence of acoustic oscillations, but this term averages to zero on timescales long compared with the sound travel time. A sketch of the interaction between the different energy reservoirs as given in equations 


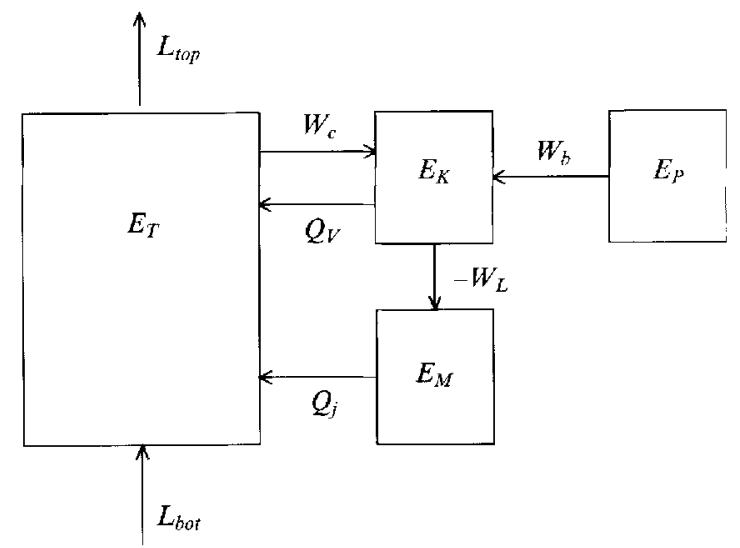

FIGURE 6. Sketch of the energy budget and coupling between the different reservoirs. Gain and loss of total energy of the system is determined by the luminosity difference between the bottom and top. The magnetic energy generated by the dynamo is supplied via the kinetic energy reservoir, which in turn is fed by thermal energy due to compression and expansion. Buoyancy work mediates an interplay between the kinetic and potential energy reservoirs.

$$
\begin{array}{lllll}
E_{M} \approx 1.3 \times 10^{-3} & -W_{L} \approx 2 \times 10^{-4} & Q_{I} \approx 2 \times 10^{-4} & \tau_{M} \approx 7 & \hat{\lambda}_{M} \approx 0.08 \\
E_{K} \approx 10^{-2} & W_{c} \approx 7 \times 10^{-4} & Q_{V} \approx 5 \times 10^{-4} & \tau_{K} \approx 20 & \hat{\lambda}_{K} \approx 0.20 \\
E_{P} \approx-1.3 & W_{b} \approx \pm 10^{-4} & & & \\
E_{T} \approx 10 & L_{b o t} \approx 5 \times 10^{-3} & L_{\text {top }} \approx 5 \times 10^{3} & \tau_{T} \approx 2 \times 10^{3} &
\end{array}
$$

TABLE 3. Estimates for the various energies and energy fluxes for the standard case with $\operatorname{Pr}_{M}=4$; Run A.

(4.1) $(4.4)$ is shown in figure 6 . Below we give rough estimates for the different terms and discuss the interactions between the different forms of energy.

Owing to dynamo action the magnetic energy increases and the kinetic energy decreases slightly until saturation occurs with $E_{M} \approx 0.1 E_{K}$. We investigate the details of the energy flow by considering simple model equations which are analogous to the original energy equations.

We neglect the potential energy and the buoyancy work terms because when taken over sufficiently long time intervals, the average value of $W_{b}$ is zero. The contents of the three remaining energy reservoirs, their decay timescales $\tau_{M}=E_{M} / Q_{J}$, $\tau_{K}=E_{K} / Q_{K}, \tau_{T}=E_{T} / L_{b o t}$ (Kelvin-Helmholtz timescale), and the various work terms are measured. The approximate results of such measurements are given in table 3. All quantities are non-dimensional, for example time is in units of $(d / g)^{1 / 2}$; see $\S 2.3$.

The evolution of the magnetic energy (figure 4) shows a maximum with a subsequent small decrease. The timescale for this decay is shorter than the magnetic diffusion timescale $d^{2} /\left(\eta \pi^{2}\right) \approx 2000$. Similar behaviour was noted by Kida, Yanase \& Mizushima (1991). If this decay persisted then the magnetic energy would decay by many orders of magnitude within one magnetic diffusion time. In order to investigate this worrying possibility we now consider simple master equations which describe the energy balance and the energy transfer between the various reservoirs.

The construction of such equations is best understood by considering the magnetic energy equation (4.1). As the dynamo saturates, the rate of working changes much 

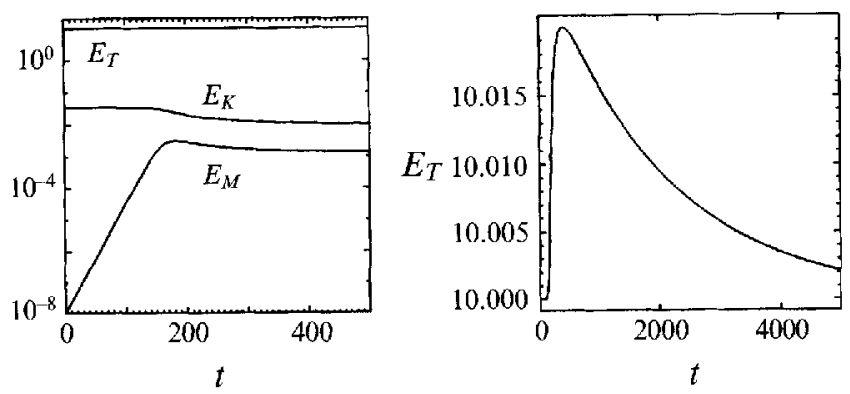

FIGURE 7. (a) Evolution of the different energy forms obtained by integrating the model equations (4.7)-(4.9). An enlarged picture of the evolution of the thermal energy is shown in $(b)$, but over a longer timescale.

less than the dissipation rates. From figure $4(b)$ we see that $\lambda_{M}$ varies only slightly with time. Therefore the ratio $\tau_{M}=E_{M} / Q_{J}=\frac{1}{10} \lambda_{M}^{2} / \eta$ does not vary much, and we may approximate the Joule dissipation in our set of equations by $E_{M} / \tau_{M}$. It is the very small difference between $W_{L}$ and $Q_{J}$ that is important here, rather than their actual values. During the growth phase of the dynamo the work done against the Lorentz force, $-W_{L}$, is slightly larger than the work done by dissipation. However, once the dynamo saturates (see figure 6 in Paper I) these work terms are approximately equal. This behaviour may be approximated by a nonlinear 'quenching' term which becomes important once the magnetic energy equals a significant fraction of the kinetic energy. For weak magnetic energies we require $-W_{L}=R_{M} E_{M} / \tau_{M}$, which is a factor $R_{M}$ larger than $Q_{J}$. Here, $R_{M}$ plays the role of a bifurcation parameter that must be larger than unity if there is to be a dynamo (see below). For large values of $E_{M}$ we require that $-W_{L} \approx Q_{J}$. The simplest ansatz is

$$
-W_{L}=R_{M} \frac{E_{M}}{\tau_{M}}\left(1-\frac{E_{M}}{\tau_{M}} / \frac{E_{K}}{\tau_{K}}\right)
$$

This quenching expression vanishes if $E_{M} / E_{K}=\tau_{M} / \tau_{K}$. The same term must enter with opposite sign in the momentum equation. For the compression work term we assume a similar form. The complete set of nonlinear equations may then be written as

$$
\begin{gathered}
\frac{\mathrm{d} E_{M}}{d t}=R_{M} \tilde{E}_{M}\left(1-\frac{\tilde{E}_{M}}{\tilde{E}_{K}}\right)-\tilde{E}_{M}, \\
\frac{\mathrm{d} E_{K}}{\mathrm{~d} t}=R_{K} \tilde{E}_{K}\left(1-\frac{\tilde{E}_{K}}{\tilde{E}_{T}}\right)-R_{M} \tilde{E}_{M}\left(1-\frac{\tilde{E}_{M}}{\tilde{E}_{K}}\right)-\tilde{E}_{K}, \\
\frac{\mathrm{d} E_{T}}{\mathrm{~d} t}=-R_{K} \tilde{E}_{K}\left(1-\frac{\tilde{E}_{K}}{\tilde{E}_{T}}\right)+L_{b o t}-\tilde{E}_{T}+\tilde{E}_{K}+\tilde{E}_{M},
\end{gathered}
$$

where a tilde denotes division by the appropriate timescale, i.e. $\tilde{E}_{M}=E_{M} / \tau_{M}$, etc. Here, $R_{K}$ is another bifurcation parameter governing the onset of convection.

The steady-state solutions of these equations are $E_{T}=\tau_{T} L_{b o t}, E_{K}=\tau_{K} L_{b o t}[1-$ $\left.R_{K}^{-1}\left(2-R_{M}^{-1}\right)\right]$, and $E_{M}=\left(\tau_{M} / \tau_{K}\right) E_{K}\left(1-R_{M}^{-1}\right)$. We have integrated these equations using the relaxation timescales found in our simulation; see table 3 . The two bifurcation parameters have been adjusted such that the three energies of the steady state correspond to those of our simulation, i.e. $R_{M}=\left[1-\left(E_{M} \tau_{K}\right) /\left(E_{K} \tau_{M}\right)\right]^{-1}=1.591$ and $R_{K}=\left(2-R_{M}^{-1}\right)\left[1-\left(E_{K} \tau_{T}\right) /\left(E_{T} \tau_{K}\right)\right]^{-1}=1.524$. The results are shown in figure 7, 
where we used the appropriate steady solution of the non-magnetic state as the initial condition.

Once the magnetic field becomes significant the kinetic energy decreases slightly. At the same time there is a small increase in the thermal energy followed by a slow relaxation to the same energy as before the onset of dynamo action. However, the thermal energy is extremely small, and such a change would be difficult to confirm in an actual dynamo simulation where there are large fluctuations. Although this simple model was only designed to describe the qualitative behaviour of a convective dynamo, it quantitatively reproduces various features, such as the dynamo's growth rate.

\subsection{Magnetic flux tubes}

A remarkable result that emerged from our simulations is the formation of large-scale, coherent, magnetic flux tube structures. These tubes are similar to vorticity tubes found in homogeneous turbulence (e.g. Kerr 1985; She, Jackson \& Orszag 1990; Vincent \& Meneguzzi 1991). In the literature several mechanisms for the formation of magnetic flux tubes have been discussed: expulsion of flux from convection cells (Galloway, Proctor \& Weiss 1977), instabilities (Parker 1979), and fragmentation of buoyant flux (Schüssler 1977; 1984).

Snapshots of the magnetic field at two different times during this amplification show that in the saturated state the magnetic tubes are longer and more clearly defined than in the growth phase (figure 8). This may be quantified by the increase of $\lambda_{M}$ during the growth phase of the dynamo; see figure $4(b)$. Thus there is a noticeable change in the flow structure as the field is amplified prior to saturation. This indicates that the formation of extended tubes is a dynamical process where the feedback between the magnetic field and the motion is important.

In order to illuminate the mechanism of flux tube formation further, we computed the kinematic evolution of the magnetic field with a steady velocity field taken from $t=t_{2}$; see figure 4. A snapshot of the magnetic field in such a simulation is given in figure 9. It shows that the growing magnetic field has the topology of flux tubes which is a consequence of the stretching and compression of the convective velocity field. The dynamical feedback of the Lorentz force seems to cause a reduction of the tubes' curvature (compare figures $8 b$ and 9 ).

\subsection{Magnetic buoyancy}

Magnetic buoyancy of flux tubes is widely believed to be responsible for transporting magnetic flux from the bulk of the convection zone up to the solar surface on a timescale much shorter than the period of the solar cycle. In our simulations this process does not seem to be very important, and instead we find downward transport of magnetic fields due to advection.

Figure 10 shows horizontal cross-sections of several variables across part of a strong magnetic flux tube. A vertical vortex tube is also present. Velocity is directed clockwise around the vortex tube. In a video animation we see a long magnetic flux tube being wound around this vortex tube.

The simulations show that flux tubes are a direct consequence of the flow field, and that downward motions around the tube are important. We do not see tubes rising under the influence of magnetic buoyancy. This could be a selection effect: owing to stratification, rising tubes expand and therefore become weaker and thus lose their identity. We see flux tubes mainly at stages where their identity is maintained by stretching and compression due to the flow. 
(a)

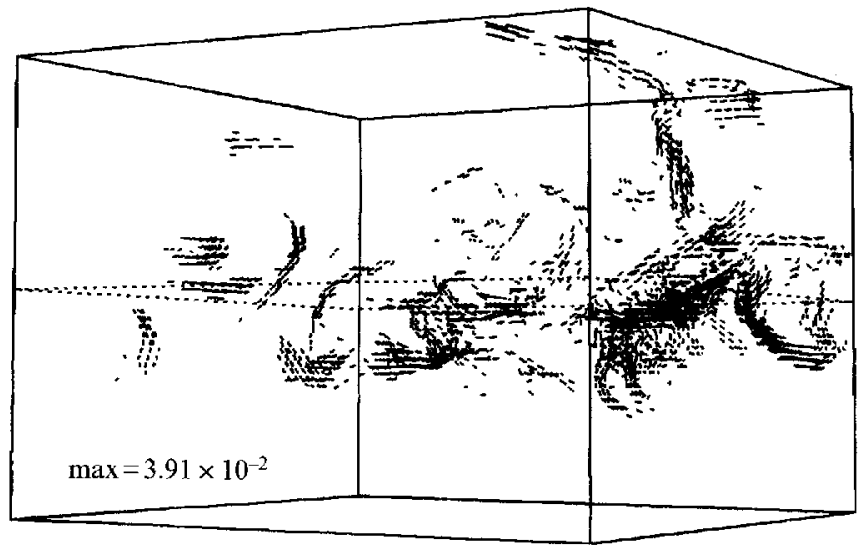

(b)

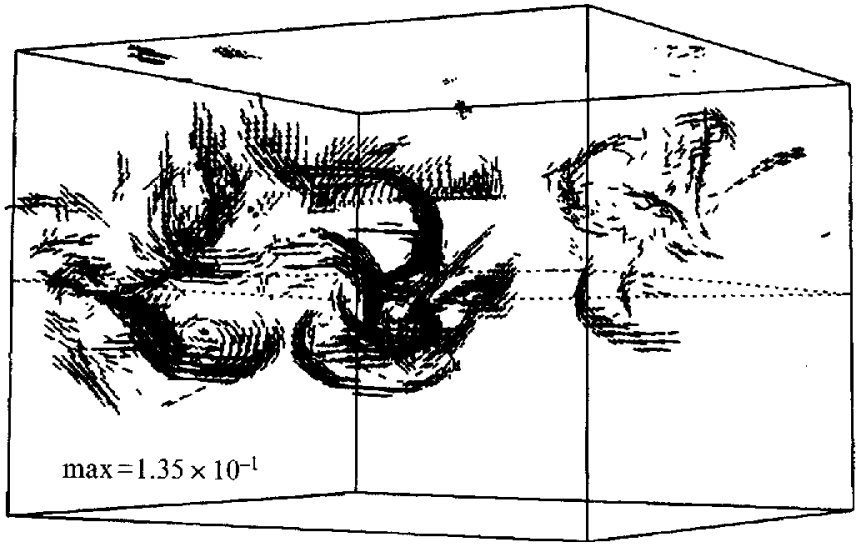

FIGURE 8. (a) Magnetic field during amplification at $t=514.7$. Only vectors above a certain threshold are plotted $(B>0.005)$. The interface between stably and unstably stratified layers is marked by a dotted line. (b) Distribution of magnetic field at a later time close to saturation at $t=653.8$. Threshold $B>0.045$. Run A.

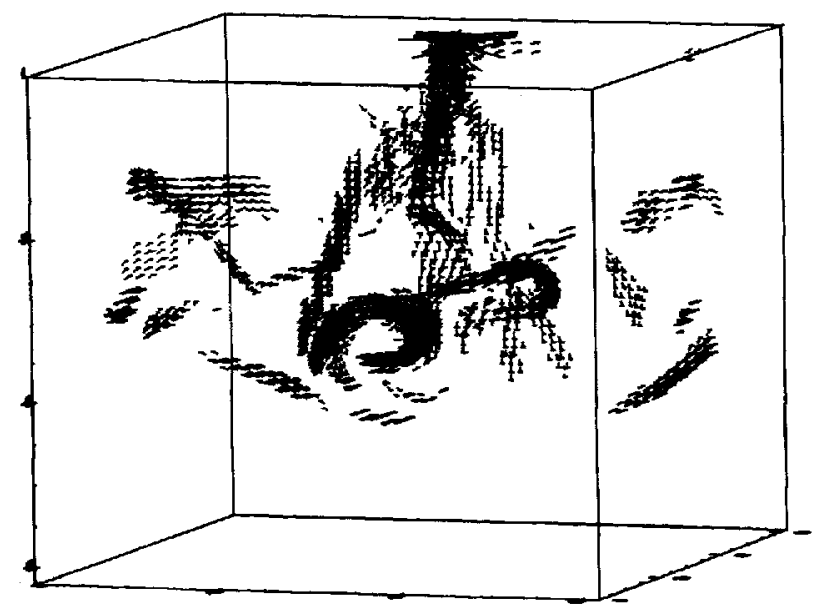

Figure 9. A snapshot for the frozen-in kinematic case at $t=856.9$. Only vectors above a certain threshold are plotted $\left(B>4 \times 10^{-4}\right)$. Run $A$. 
We next consider the forces acting on the tube. By examining the cross-sections of density, and gas and magnetic pressures we find that the influence of the magnetic field on the density, which results in magnetic buoyancy, is weaker than the drag force of the velocity field. The tube's magnetic curvature force, in this case, is in the horizontal direction and is therefore irrelevant in determining its vertical acceleration.

The relative density reduction $\Delta \rho / \rho$ in the strongest horizontal tubes is about $3 \%$ (see figure 10), approximately equal to the ratio of magnetic to gas pressure, i.e. $\Delta \rho / \rho \approx \boldsymbol{B}^{2} /\left(2 \mu_{0} p\right)$. The acceleration $a$ of the tube is expected to be approximately $g \Delta \rho / \rho=0.03$. Figure $10(b)$ shows the profile of the vertical velocity along the line marked in the contour plots. Surrounding the tube there is a downflow whose speed is about 0.1 velocity units. However, within the tube this downflow is reduced to 0.02 due to the buoyancy of the magnetic flux tube. The velocity of the tube relative to the surrounding medium is therefore $\Delta u=0.08$. The duration of this acceleration relative to the surroundings is $\Delta t=\Delta u / a \approx 3$, which is comparable with the typical lifetime of these tubes. The displacement associated with this acceleration is $\Delta z=\frac{1}{2} a \Delta t^{2} \approx 0.1$, showing that the tubes are not advected far during their lifetime. The lifetime of such strong tubes is typically twice as long as the duration of their advection, but this includes the time for its generation and disappearance. The tubes disappear by ohmic diffusion, i.e. on a diffusion timescale based on the magnetic Taylor microscale, $\tau_{M}$.

In a video animation one occasionally sees rapid downward motions of flux tubes surrounding intense downdraughts, but these tubes are more vertically oriented than the tube considered in figure 10. An example of such a tube may be seen in figure $8(b)$. Vertically oriented flux tubes are often found in the upper part of the convection zone, whilst close to the interface, tubes are usually oriented horizontally.

In our simulations magnetic buoyancy is counterbalanced by drag forces. It is an open question, however, how far these conclusions carry over to the solar case, where the Mach numbers at the bottom of the convection zone are expected to be much smaller $\left(\sim 10^{-3}\right)$ than in our simulations $(\gtrsim 0.1)$, and where much stronger toroidal magnetic fields can be generated by differential rotation.

\subsection{Magnetic field stratification}

In a two-dimensional simulation in the $(x, z)$-plane with an imposed magnetic flux in the $y$-direction Jennings et al. (1992) found that in the relaxed state the quantity $\langle\boldsymbol{B}\rangle /\langle\rho\rangle$ is almost uniformly stratified throughout the convection zone, and that $\langle\boldsymbol{B}\rangle /\langle\rho\rangle$ goes rapidly to zero in the overshoot layer. In their simulation the total magnetic flux is conserved and non-vanishing, whereas in the present case the total flux vanishes. We therefore investigated the variation of $B_{r m s}=\left\langle\boldsymbol{B}^{2}\right\rangle^{1 / 2}$ which increases towards the upper layers (figure 11).

For comparison, we have also shown both the depth dependence of $B_{r m s}$ and the (non-dimensional) average Alfvén speed $v_{\boldsymbol{A}}=\left\langle\boldsymbol{B}^{2} / \rho\right\rangle^{1 / 2}$. In the bulk of the convection zone the r.m.s. value of the magnetic field increases downwards and has a maximum at the interface. However, the average Alfvén speed varies little through the convection zone.

\section{Statistics}

In the previous section we characterized the onset and the energy budget of turbulent convective dynamos. The magnetic field is always in the form of magnetic flux tubes. In this section we focus on the question of how these tubes are oriented 
(a)

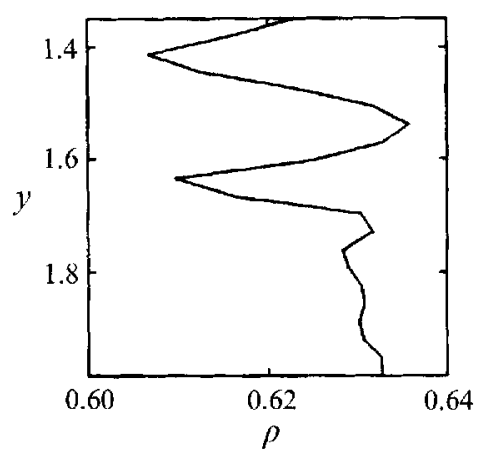

(b)

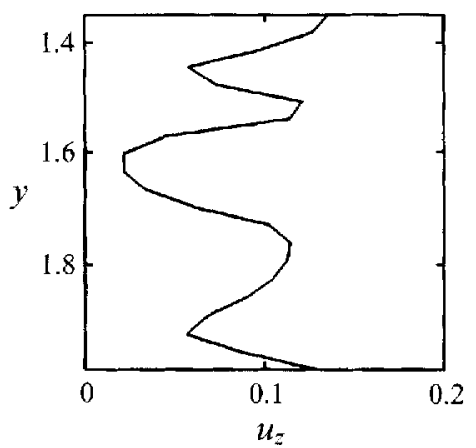

(c)

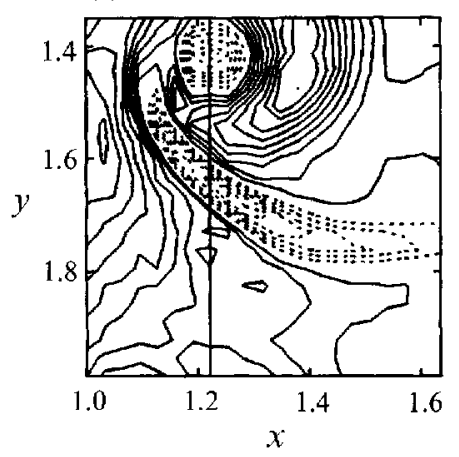

(d)

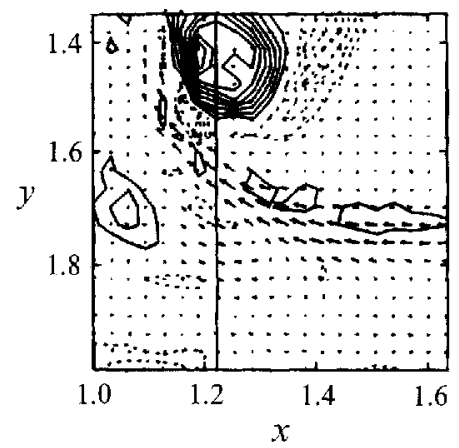

FIgure 10. Horizontal cross-section $(z=0.68)$ through a strong magnetic flux tube. Plots $(c)$ and (d) show the density fluctuation and the vertical vorticity component (dotted line means negative values). The magnetic flux tube is shown as vectors superimposed on $(d)$. Note that the flux tube is wrapped around the vortex tube. Plots $(a)$ and $(b)$ show profiles of the density and the vertical velocity component through the vertical line indicated in $(c)$ and $(d)$. Positive values of $u_{z}$ mean downward velocity. Note that $u_{z}$ is small, but positive in the core of the magnetic flux tube, i.e. buoyancy counteracts the downward motion, but is not sufficient to lift the tube. Run A.

(a)

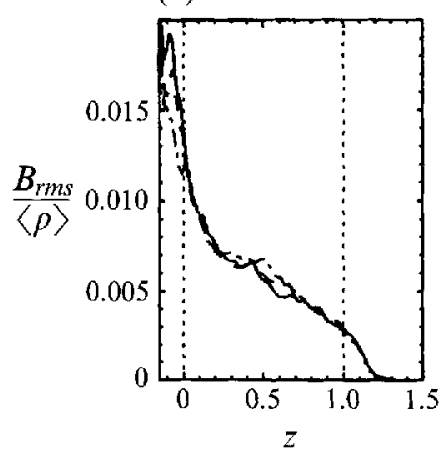

(b)

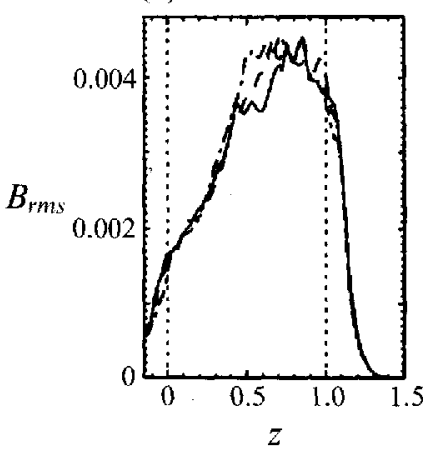

(c)

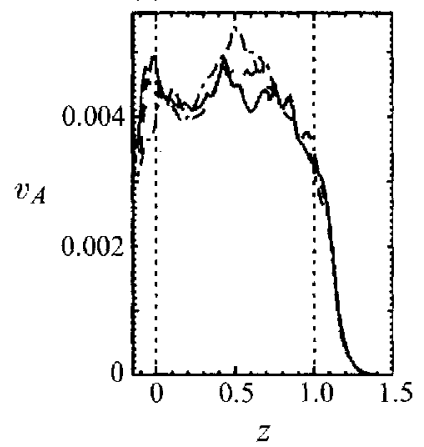

FIGURE 11. (a) Vertical dependence of $B_{r m s} /\langle\rho\rangle$, where $B_{r m s}=\left\langle B^{2}\right\rangle^{1 / 2}$. Note that this quantity increases slightly towards the upper layers $(z=0)$. $(b)$ Vertical dependence of $B_{r m s}$ on $z$. In the convection zone this quantity increases with depth. Note the peak of $B_{r m s}$ near the interface $(z=1)$. (c) Depth dependence of the average Alfvén speed $v_{A}=\left\langle\boldsymbol{B}^{2} / \rho\right\rangle^{1 / 2}$. Run D. 

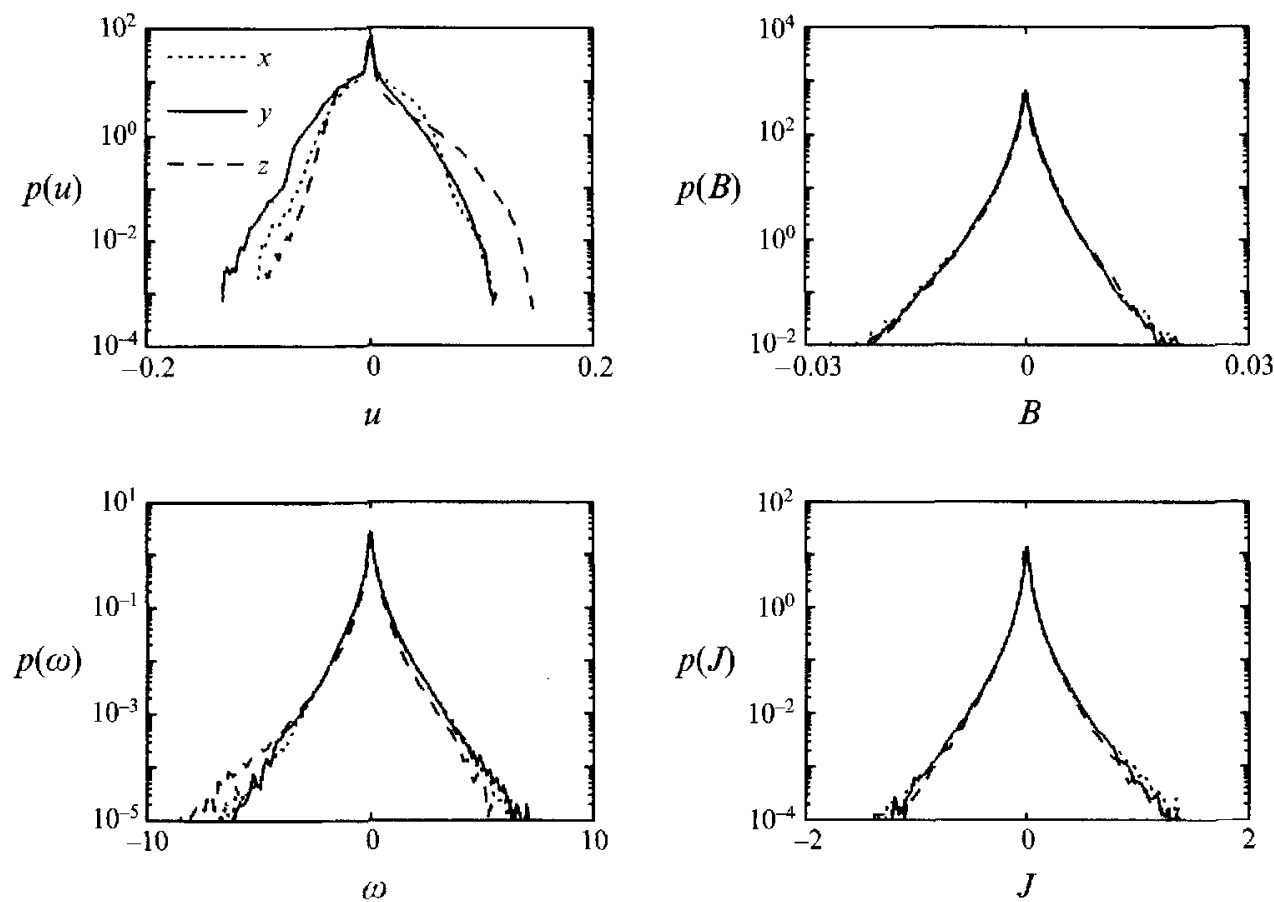

FiguRE 12. The probability density functions of the three components of $\boldsymbol{u}, \boldsymbol{\omega}, \boldsymbol{B}$, and $\boldsymbol{J}$. Note the strong asymmetry of the $u_{z}$ field showing an extended range of strong downdraught motions $\left(u_{z}>0\right)$. There is also a slight asymmetry in the distribution of the vertical vorticity with a somewhat enhanced probability of $\omega_{z}>0$ associated with the vorticity generated in downdraughts. There are no marked asymmetries in the distributions of $\boldsymbol{B}$ and $\boldsymbol{J}$. Run D.

relative to other relevant fields in the simulation. For this purpose we employ statistical tools such as probability density functions.

\subsection{Probability density functions}

In figure 12 we present probability density functions (PDFs) of $\boldsymbol{u}, \boldsymbol{\omega}, \boldsymbol{B}$, and $\boldsymbol{J}$. The PDFs have extended exponential tails. Note that the distribution of $B$ is more peaked than that of $u$. This shows that $\boldsymbol{B}$ is more intermittent than $\boldsymbol{u}$. Similarly peaked distribution functions for the magnetic field have been obtained by Dittrich et al. (1988).

The exponential tails of the PDFs of transverse velocity gradients are associated with intermittency in hydrodynamic turbulence (Kraichnan 1990), and have also been found in other numerical simulations (e.g. Vincent \& Meneguzzi 1991). Such exponential tails typiçally occur with highly turbulent convection (e.g. Castaing et al. 1989), although they can also occur in non-turbulent flows (Pumir, Shraiman \& Siggia 1991).

The degree of intermittency of a function $f$ is characterized by its kurtosis $\operatorname{kurt}(f)=$ $\left\langle f^{4}\right\rangle /\left\langle f^{2}\right\rangle^{2}$. For Run D, we found kurtosis values around $4-5$ for the horizontal velocity components and 8 for the vertical component. The kurtosis of the other fields is much larger (around 20 for the vorticity and the magnetic field and around 30 for the electric current $\boldsymbol{J}$ ). These values are large compared with the Gaussian distribution's value of 3 , which indicates a high degree of intermittency. 
(a)

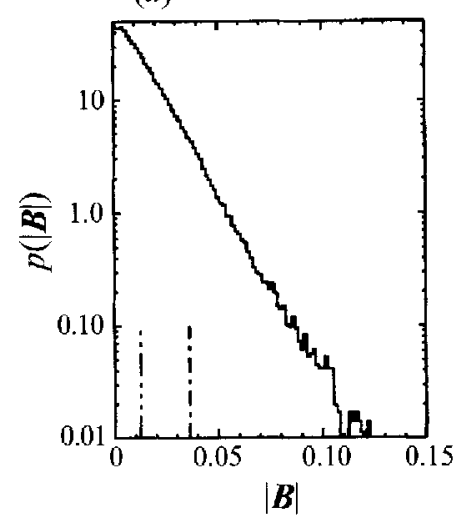

(b)

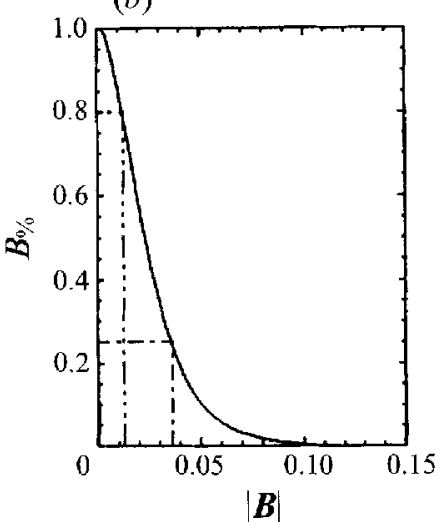

$(c)$

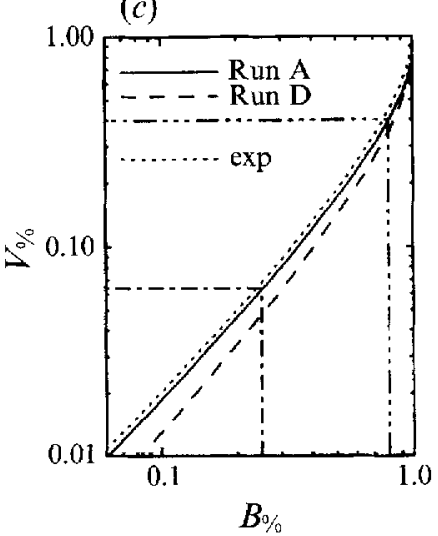

Figure 13. (a) PDF of $|\boldsymbol{B}|$. (b) The fraction $B_{\%}$ of the magnetic field for different values of the threshold $|\boldsymbol{B}|$. (c) The minimum fractional volume $V_{\%}$ occupied by the regions in space that contribute a fraction $B_{\%}$ to the magnetic field. Here, $B_{1}=B_{r m s}$ and $B_{2}=\frac{1}{4} B_{\max }$ are used for abbreviation. Run A. In $(c)$ we compare data from Run D and the relation (5.2), referred to as "exp”.

\subsection{Filling factor}

An interesting quantity that can be derived from the PDF of $|\boldsymbol{B}|$ is the smallest percentage of the volume $V_{\%}$ occupied by a certain percentage $B_{\%}$ of the total field. The relation $V_{\%}\left(B_{\%}\right)$ may be expressed in terms of $p(B)$ by

$$
V_{\%}=\int_{B^{*}}^{\infty} p(|\boldsymbol{B}|) \mathrm{d}|\boldsymbol{B}| \quad \text { and } \quad B_{\%}=\langle|\boldsymbol{B}|\rangle^{-1} \int_{B^{*}}^{\infty}|\boldsymbol{B}| p(|\boldsymbol{B}|) \mathrm{d}|\boldsymbol{B}|,
$$

see figure 13 . We see, for example, that magnetic fields which exceed their r.m.s. value make up $80 \%$ of the field (figure $13 \mathrm{~b}$ ) and are contained in only $40 \%$ of the volume (figure $13 \mathrm{c}$ ). Further, the field vectors with $|\boldsymbol{B}| \geqslant \frac{1}{4} B_{\max }$ contribute $25 \%$ to the total field and occupy only $5 \%$ of the volume.

The relation $B_{\%}$ versus $V_{\%}$ is not significantly resolution dependent. This is because the PDF of $|\boldsymbol{B}|$ is nearly exponential. If it was exactly exponential, the relation would be

$$
B_{\%}=\left(1-\ln V_{\%}\right) V_{\%},
$$

which is shown in figure 13 for comparison.

\subsection{Correlations}

It has often been emphasized that the equations for vorticity and magnetic field are very similar (e.g. Batchelor 1950). It is therefore interesting to compare these two vector fields. How certain vectors are aligned with each other cannot easily be determined by looking at cross-sections. Only a statistical analysis yields quantitative trends.

In figure 14 we present histograms of the cosines of the angles between the different vector fields. There is indeed a high probability for $\boldsymbol{\omega}$ and $\boldsymbol{B}$ to be either parallel or antiparallel, especially when the magnetic field is strong. In a video animation we observed however that strong vortex tubes and strong magnetic fields avoid each other. Yet, when $|\boldsymbol{B}|$ is strong the two vectors are aligned. This suggests that strong magnetic fields enslave weaker vorticity.

The cosines of the angles between all other fields appear to be random. However, it 

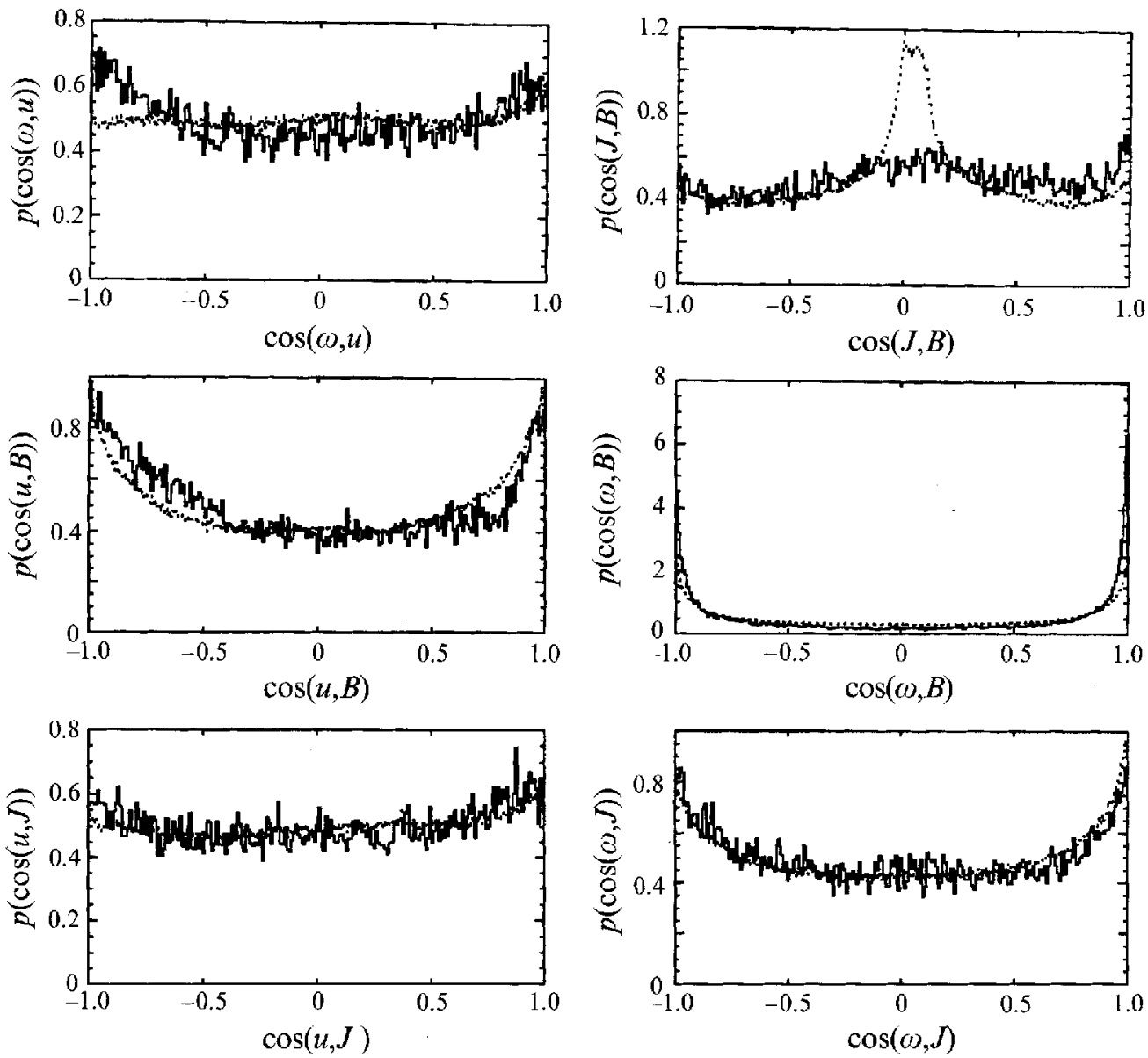

Figure 14. Probability density functions of the cosines of the angles between different vector fields. Solid lines refer to points where $|\boldsymbol{B}| \geqslant 3 B_{r m s}$, and dotted lines are for all points. Note the strong alignment between $\boldsymbol{\omega}$ and $\boldsymbol{B}$. Run D.

is evident that $\boldsymbol{J}$ and $\boldsymbol{B}$ are predominantly perpendicular when the field is weak, and that this changes markedly when the field is strong. This must be due to the saturation of the dynamo: as the field becomes strong, $\boldsymbol{J}$ and $\boldsymbol{B}$ are no longer perpendicular, the work done against the Lorentz force, $-\langle\boldsymbol{u} \cdot(\boldsymbol{J} \times \boldsymbol{B})\rangle$, is lowered and the conversion of kinetic to magnetic energy is reduced.

A similar selective analysis, but for the hydrodynamic case, has been presented by Pelz et al. (1985) and Kerr (1987), who used the local dissipation as a selection criterion. They found large helicity densities in regions of low dissipation, and more uniform distributions in regions of high dissipation.

\subsection{Eigenvectors of the rate of strain tensor}

The flow structure may be analysed using the eigenvectors of the rate of strain tensor $s_{i j}=\frac{1}{2}\left(u_{i, j}+u_{j, i}\right)$. This symmetric $3 \times 3$ matrix is computed at each mesh point. The eigenvectors $\boldsymbol{e}_{1}, \boldsymbol{e}_{2}, \boldsymbol{e}_{3}$ are ordered such that the corresponding (real) eigenvalues satisfy $\lambda_{1}<\lambda_{2}<\lambda_{3}$. The sum of the eigenvalues would be exactly zero for an incompressible fluid, and here the sum is small compared to $\max \left(\lambda_{i}\right)$. This is consistent with the fact that $\left\langle(\operatorname{div} u)^{2}\right\rangle \ll\left\langle\omega^{2}\right\rangle$, indicating that compressibility effects are weak on average. 

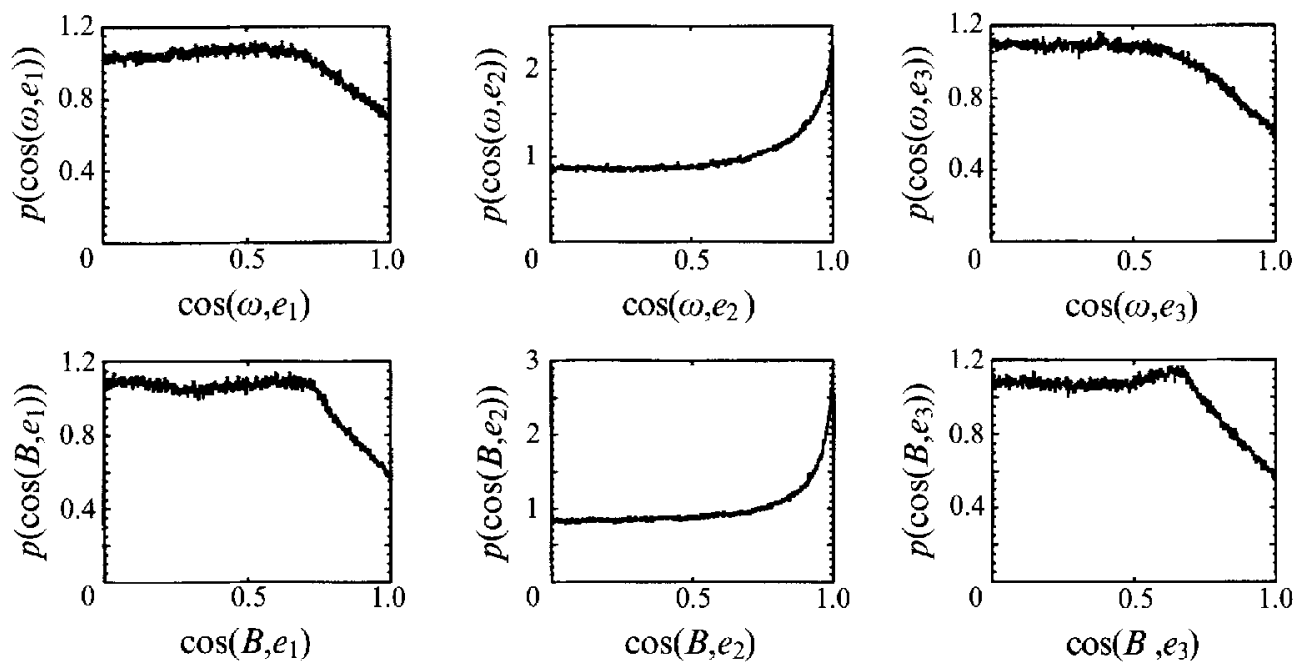

Figure 15. Correlations between the three eigenvectors of the rate of strain tensor and vorticity (upper row) and magnetic field (lower row). Run D.

The three eigenvectors are orthogonal, with $\boldsymbol{e}_{1}$ giving the direction of compression and $e_{3}$ the direction of stretching. If $\lambda_{2}=0$ the flow is two-dimensional. In practice, $\lambda_{2}$ is usually small compared with $\lambda_{1}$ and $\lambda_{3}$.

In figure 15 we show the probability density functions of the cosines of the angles between the three eigenvectors of the rate of strain tensor and both the vorticity and the magnetic field. Note that there is an enhanced correlation between $e_{2}$ and both the vorticity and the magnetic fields. There is also some tendency for $\boldsymbol{\omega}$ and $\boldsymbol{B}$ to be perpendicular to $\boldsymbol{e}_{1}$ and $\boldsymbol{e}_{3}$, the eigenvectors corresponding to the directions of compression and stretching, respectively. We have seen before that the probability for vorticity and magnetic field to be aligned is indeed larger than the probability for them to be perpendicular. Similar relations between vorticity and the three eigenvectors have been observed in the simulations of homogeneous turbulence by Kerr (1985), and Vincent \& Meneguzzi (1991). These authors explain the correlation between vorticity and the intermediate eigenvector as an indication of the quasi-two-dimensionality of the flow surrounding vortex tubes.

It may seem somewhat surprising that there is no alignment of $\boldsymbol{\omega}$ or $\boldsymbol{B}$ with $\boldsymbol{e}_{3}$ (the eigenvector corresponding to the direction of stretching), because vortex stretching and magnetic field line stretching are known to be important properties of turbulence and dynamos, respectively. Stretching does, however, contribute to the dynamics of vorticity and magnetic fields in that it causes the tubes to have non-circular crosssections. This can be seen in figure 16 where we compare the directions of the eigenvectors $\boldsymbol{e}_{1}$ and $\boldsymbol{e}_{3}$ with the position of a strong magnetic flux tube in a vertical slice through the plane $x=1.4$. This flux tube is the same as that in the horizontal slice of figure 10. In vertical section the velocity is mainly downwards, but in the immediate vicinity of the tube, $u_{z}$ is very small; see $\S 4.4$. The eigenvectors $\boldsymbol{e}_{1}$ and $\boldsymbol{e}_{3}$ show that the tube is compressed in the vertical direction, but stretched in the horizontal $y$-direction. As a consequence the tube is flat.

The statistical investigations presented in this section have shown that the magnetic field vector has a high probability to be aligned both with the vorticity and the intermediate eigenvector of the rate of strain matrix. This supports the anticipated similarity between the magnetic field and the vorticity. 

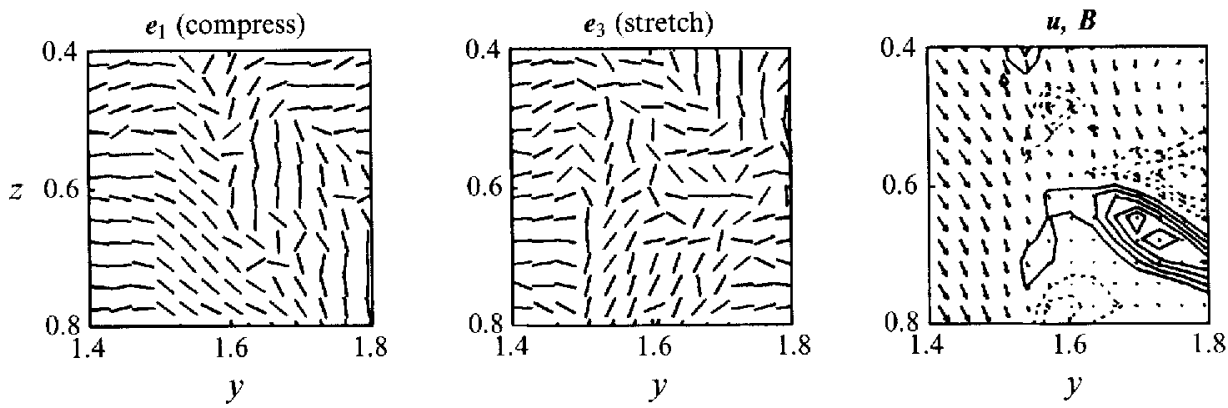

Figure 16. Vertical cross-section through the plane $x=1.4$ perpendicular to a strong magnetic flux tube. Projections of the eigenvectors fields $e_{1}$ and $e_{3}$ are shown together with contours of $B_{x}$ and vectors $\left(u_{y}, u_{z}\right)$. Solid (dotted) contours represent negative (positive) values of $B_{x}$. Note the close proximity of positive and negative contours, which suggests that reconnection is imminent. In the surroundings of the magnetic flux tube the direction of compression, $\boldsymbol{e}_{1}$, is vertical, whereas the direction of stretching, $\boldsymbol{e}_{3}$, is horizontal. This explains the flat shape of the tube. Run A.

\section{Spectral properties}

In $\S 5$ we demonstrated that the statistical properties of magnetic and velocity structures found in our dynamo simulation are similar to those of simulations of homogeneous turbulence. We now focus attention on the spectral properties of velocity and magnetic fields.

\subsection{Energy and helicity spectra}

We consider the spectra of kinetic and magnetic energies in a horizontal plane at the interface $(z=1)$ as a function of the horizontal wave vector $\left|\boldsymbol{k}_{H}\right|$, where $\boldsymbol{k}_{H}^{2}=k_{x}^{2}+k_{y}^{2}$ and write $k=\left|\boldsymbol{k}_{H}\right|$ for the length of the horizontal wave vector. We do not include the dependence on the vertical wavenumber $k_{z}$ because of the strong inhomogeneity in that direction. The spectral kinetic energy is defined as

$$
E_{K}(k)=\frac{1}{2}\langle\rho\rangle \int_{0}^{2 \pi}\left|\hat{\boldsymbol{u}}\left(\boldsymbol{k}_{H}, z, t\right)\right|^{2} k \mathrm{~d} \phi_{k},
$$

where $\phi_{k}=\arctan \left(k_{y} / k_{x}\right)$ and the hat denotes the Fourier transformation of the three components of $\boldsymbol{u}$. Likewise, the spectral magnetic energy is defined as

$$
E_{M}(k)=\frac{1}{2} \int_{0}^{2 \pi}\left|\hat{\boldsymbol{B}}\left(\boldsymbol{k}_{H}, z, t\right)\right|^{2} k \mathrm{~d} \phi_{k} .
$$

In order to investigate the spectral properties of the effective 'forcing term' we also compute the spectrum of the temperature fluctuations $\Theta=e-\langle e\rangle$

$$
E_{\Theta}(k)=\int_{0}^{2 \pi}\left|\hat{\Theta}\left(\boldsymbol{k}_{H}, z, t\right)\right|^{2} k \mathrm{~d} \phi_{k} .
$$

We compare the spectra of the growth and saturation phases of the dynamo; see figure 17. In both cases the kinetic energy spectrum shows a short inertial range with a slope compatible with $E_{K}(k) \sim k^{-5 / 3}$ followed by an extended dissipation range. Obviously, the inertial range in our simulation is too short to accurately determine the slope. Thus, our data are equally compatible with a $k^{-2}$ spectrum.

During the growth phase of the dynamo the magnetic energy spectrum is different from that of the saturated state. In the growth phase at $t=t_{1}$ there is an inertial range with a slope compatible with $k^{+1 / 3}$. This was predicted by Moffatt (1961) 

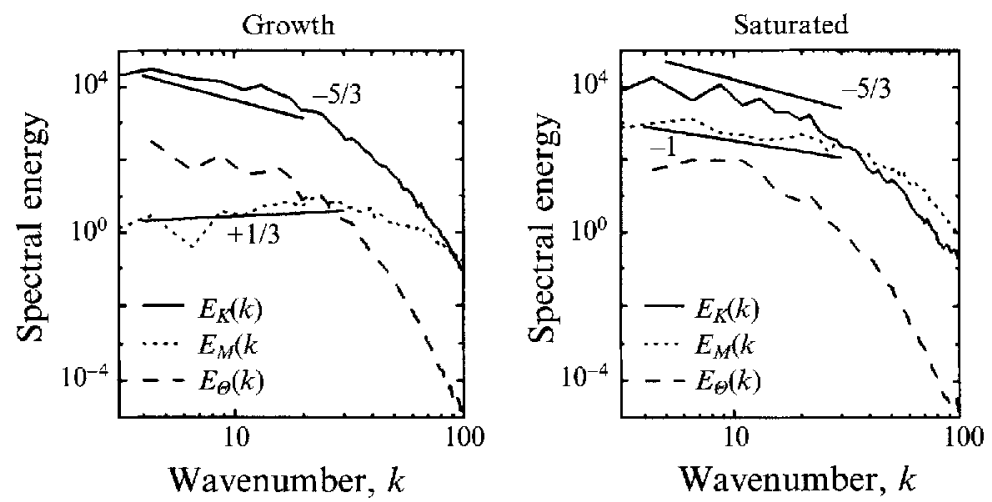

FIGURE 17. Kinetic, magnetic and thermal energy spectra during the growth phase $\left(t=t_{1}\right)$ and the saturated phase $\left(t=t_{2}\right)$ taken at the interface $(z=1)$. (The times are the same as in figure 4.) The spectra are integrated over shells $\left|\boldsymbol{k}_{H}\right|=k$. Run A.

(a)

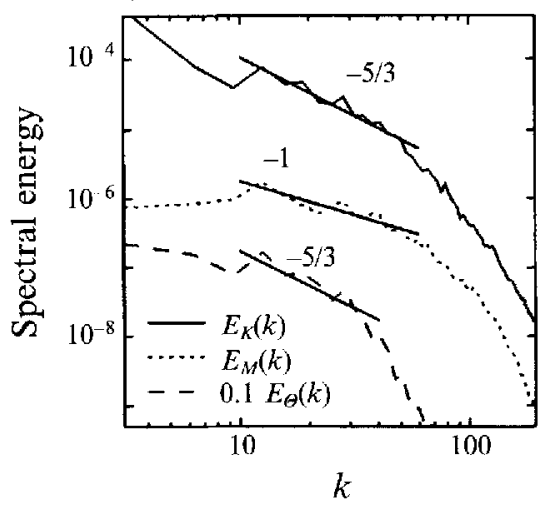

(b)

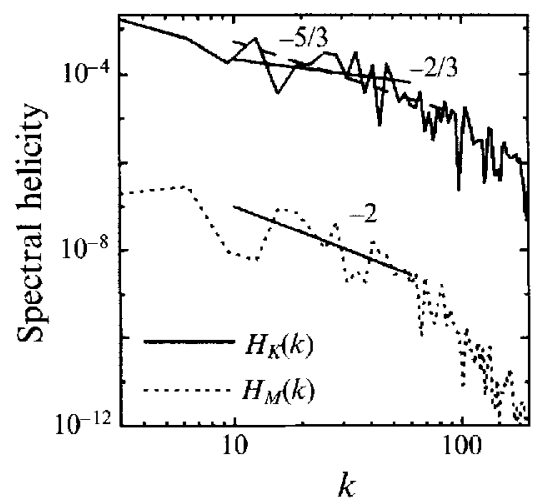

FIGURE 18. (a) Kinetic, magnetic and thermal energy spectra and (b) kinetic and magnetic helicity spectra, i.e. $\left|\hat{\omega} \cdot \hat{\boldsymbol{u}}^{*}\right|$ and $\left|\hat{\boldsymbol{A}} \cdot \hat{\boldsymbol{B}}^{*}\right|$, at $z=1$. The spectra are integrated over shells $\left|\boldsymbol{k}_{H}\right|=k$. Run $\mathrm{D}$.

using the analogy between vorticity and magnetic field. During the saturated state at $t=t_{2}$ the inertial range of the magnetic energy spectrum has a slope compatible with $k^{-1}$ (Ruzmaikin \& Shukurov 1982), but a $k^{-3 / 2}$ spectrum (Kraichnan 1965) is also compatible with the data. In figure 18 we give the spectra for a higher resolution case (Run D) for the saturated phase and we see that here too the kinetic and magnetic energy spectra have an intermediate range close to $k^{-5 / 3}$ and $k^{-1}$, respectively.

At small scales, the magnetic energy exceeds the kinetic energy in Run A, where $\operatorname{Pr}_{M}=4$. In Run D, where $\operatorname{Pr}_{M}=0.5$, the magnetic energy saturates at smaller values than the kinetic energy. This demonstrates that the saturation value of the magnetic field cannot simply be estimated using global equipartition arguments. Similarly we should note that in the presence of shear, magnetic energies in excess of the kinetic energy of the turbulence are also possible, as was recently demonstrated in a simulation by Brandenburg et al. (1995a).

Kida et al. (1991) obtained a $k^{-2}$ kinetic energy spectrum from their simulations of forced MHD turbulence and a $k^{0}$ (flat) magnetic energy spectrum in the inertial range. Thus, their magnetic energy spectrum has the same slope as the enstrophy (squared vorticity) spectrum, $k^{2} E_{K}(k)$. This supports the analogy between vorticity 
and magnetic field. In our simulations the magnetic and enstrophy spectra are similar during the growth phase of the dynamo $\left(t=t_{1}\right)$, but not in the saturated phase $\left(t=t_{2}\right)$. This is in contrast to the similarity in the alignment of the two fields found in $\S 5$, that was valid both for weak and strong (dynamo saturated) magnetic fields.

Using second-order closure calculations, Pouquet, Frisch \& Léorat (1976) found that the magnetic helicity exhibits an inverse cascade which causes a growth of the magnetic field at large scales. By comparing the two panels of figure 17 we see that magnetic energy initially builds up in the small scales, but is then transferred to larger scales. This supports the idea of an inverse cascade. The model of Pouquet et al. predicts a $k^{-2}$ spectrum for the magnetic helicity, $\langle\boldsymbol{A} \cdot \boldsymbol{B}\rangle$. Our data are in rough agreement with this, see figure 18 , where kinetic and magnetic helicity spectra are also plotted.

The kinetic helicity spectrum (figure 18) displays rather large fluctuations, which makes it difficult to decide whether it is closer to $k^{-5 / 3}$, as found by André \& Lesieur (1977) in EDQNM simulation of decaying isotropic turbulence, or to $k^{-2 / 3}$, as suggested by dimensional analysis. The latter corresponds to a pure helicity cascade towards large wavenumbers (Lesieur 1990 and references therein).

\subsection{Wave excitation}

In a video animation we saw strong isolated downdraughts oscillate such that the upper part of the downdraught remained nearly fixed in space, but the lower part shook to and fro. This is suggestive of gravity wave excitation in the lower overshoot layer. Similar behaviour was observed in two-dimensional simulations with overshoot; see Hurlburt et al. (1986).

The dispersion relation in a weakly subadiabatic isothermal layer is, for small wavenumbers, $\omega=\gamma H_{p} N k$, where $H_{p}$ is the pressure scale height, $N=\left(-\boldsymbol{g} \cdot \nabla s / c_{p}\right)^{1 / 2}$ the Brunt-Väisälä frequency, and $\omega$ the wave frequency (not to be confused with vorticity). Gravity waves are evanescent in the unstable region, but their amplitude is still significant at the interface. Since the fluid in our simulation is not isothermal the Brunt-Väisälä frequency has to be replaced by some 'effective' value (this is an eigenvalue problem). In the present situation where there is a relatively strong magnetic field we may also expect the generation of Alfvén waves whose dispersion relation would be $\omega=v_{A} k$ if the coupling to other waves is ignored.

In order to study wave phenomena in our simulation we Fourier analysed the velocity with respect to $k$ and $\omega$. In figure 19 we show the $k$ and $\omega$ dependence of $|\hat{\boldsymbol{u}}(k, \omega)|^{2}$. We note that the wavenumber dependence of the kinetic energy does not vary with frequency (figure 19a). This is in contrast to the frequency dependence which shows quite different behaviour at different wavenumbers (figure 19b). Thus, one would not expect the Taylor hypothesis, i.e. an equivalence of $k$ and $\omega$ spectra, to be valid in our case.

In figure $19(c)$ we present a contour plot of $|\hat{\boldsymbol{u}}(k, \omega)|$ in the $(k, \omega)$-plane. In this diagram the change of structure along $\omega \approx(0.1-0.2) k$ is most likely due to gravity waves. The expected slope for Alfvén waves would be $\omega / k=v_{A} \approx 0.02$ (see figure 11) which is much smaller than the slope observed. A more thorough investigation of wave excitation would certainly be worthwhile, but goes beyond the scope of this paper.

\subsection{The autocorrelation function}

Before closing the section on spectral properties we investigate the autocorrelation functions $C_{u}$ and $C_{B}$, i.e. the Fourier transform of the corresponding power spectra; see figure 20. Zeldovich et al. (1987) discussed the autocorrelation function for the 
(a)

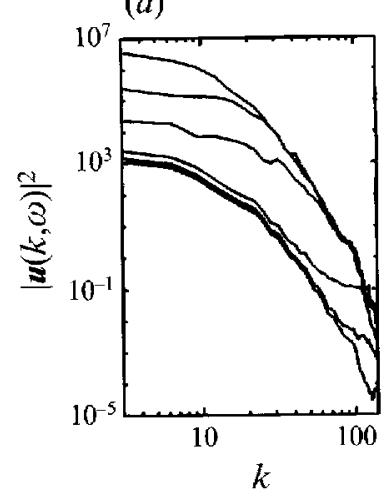

(b)

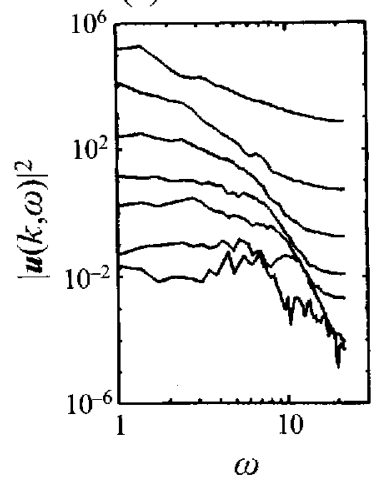

(c) $|\boldsymbol{u}(k, \omega)|^{2}$

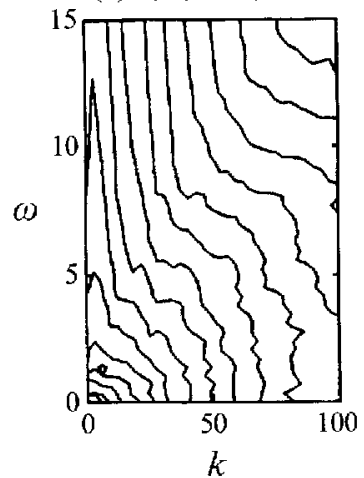

FIGURE 19. Differcnt representations of the kinetic energy spectrum $|\boldsymbol{u}(k, \omega)|^{2}$. (a) Spectrum as a function of $k$ for six different values of $\omega$. (b) Spectrum as a function of $\omega$ for seven different values of $k$. (c) Contours of the spectrum in the $(k, \omega)$-plane, which shows structure along the line $\omega \approx 0.1 k$. This structure may be related to gravity waves excited in the stably stratified region. $\Lambda$ ll spectra are computed at the interface $(z=1)$. Run A.
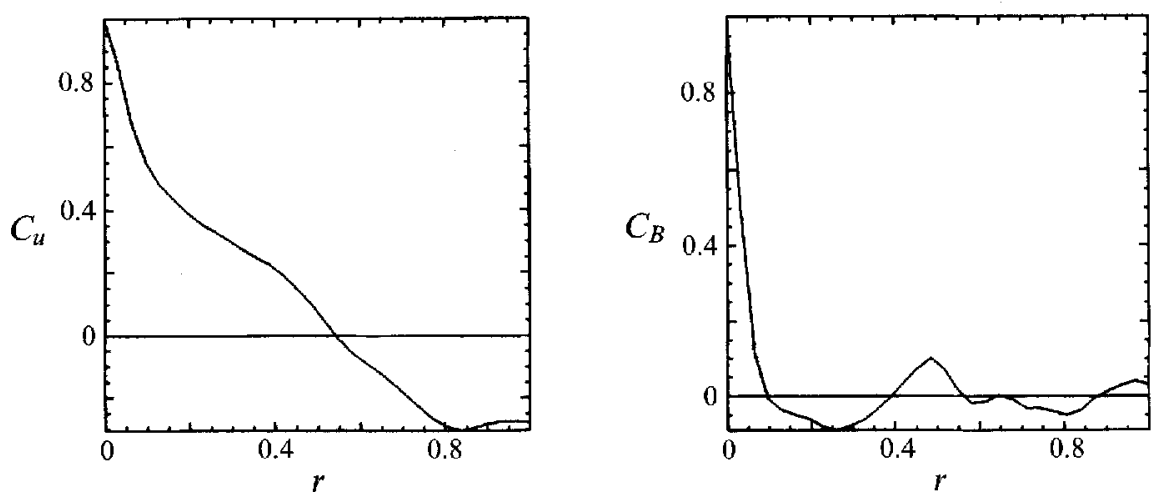

FIGURE 20. Autocorrelation functions for the velocity and magnetic field in the plane $z=1$, where $r$ is a lengthscale in the $(x, y)$-plane. Run D.

magnetic field and proposed interpretations for two lengthscales that occur in the stretch-twist-fold dynamo: the thickness of the tube, and their curvature radius; see also Zeldovich, Ruzmaikin \& Sokoloff (1990). They proposed that the width of the peak of $C_{B}$ is related to the thickness of tubes, and that the interval where $C_{B}$ is negative reflects the range of curvature radii.

For Run D the widths of the peaks of $C_{u}$ and $C_{B}$ are 0.2 and 0.05 , respectively, which is about twice the corresponding Taylor microscale. $C_{B}$ is negative in the range $0.1<r<0.4$, consistent with the visual estimates of curvature radii from plots such as figure 8 . We note that independent statistical estimates in terms of the coherence in the direction of the magnetic field (Brandenburg, Procaccia \& Segel 1995b) give similar lengthscales for the tube width and the curvature radius.

\section{Discussion}

In this paper we have carried out a detailed investigation of the properties of the magnetic field generated by dynamo action in hydromagnetic convection. A number of physical effects that are thought to be important for the solar dynamo have been 
taken into account, such as rotation and convective overshoot into the stably stratified radiative interior. Yet, we are still far from real solar conditions since we cannot use realistic values of $\eta$ and $v$, as the resulting small scales cannot be handled in a simulation such as this. This is basically a matter of spatial resolution, which also limits the maximum stratification attainable. Further limitations of our model include the relatively large values of the radiative flux and the large value of the Mach number resulting from the low sound speed. This may affect our conclusions concerning the minor role of magnetic buoyancy in our simulations. Additionally the assumption of a perfectly conducting upper boundary is not very realistic, although our model was not intended to reproduce surface conditions. Since no magnetic flux can escape from the computed domain, Petrovay (1991) has argued that such a boundary condition does not tell us whether or not magnetic buoyancy affects the dynamo. However, Parker (1984) argues that very little magnetic flux actually passes through the solar surface and thus a perfectly conducting upper boundary condition might not be too bad after all.

Despite the above limitations the model has provided us with many useful insights. Of primary importance is the fact that the flow is capable of amplifying a seed magnetic field. In other words this is an example of a self-consistent dynamo.

Another important idea to come from this work is the association of strong vorticity in areas of descending flow. As is now well known in compressible convection, there is an up/down asymmetry in which fluid descends rapidly in narrow plumes and rises more slowly in broad upwellings. Here, however, with the inclusion of rotation, descending flow acquires vorticity and swirls downwards rather than simply falling straight down. This leads to a density inversion in the core of downdraughts. Magnetic flux tubes, which come into the vicinity of these vortex tubes, seem to be amplified as they are wound round the vortex tubes. The possibility of convective overshoot allows the accumulation and storage of magnetic field in the overshoot layer. It has been argued that this is an important ingredient of the solar dynamo. Nevertheless, we should emphasize that although the dynamo actually works in the entire convection zone, owing to effective downward transport, the magnetic field is strongest at the interface between the convection zone and the radiative interior.

We find partial evidence for a similarity between vorticity and magnetic field in that vortex tubes and magnetic flux tubes are typically parallel to the intermediate eigenvector of the local rate of strain tensor, meaning that they are perpendicular to the plane in which the flow is locally two-dimensional. Such behaviour has previously been documented for vortex tubes in ordinary hydrodynamic turbulence. Furthermore, vorticity and magnetic field are parallel or antiparallel, especially in those regions where the magnetic field is strong.

During the growth phase of the dynamo the power spectra of enstrophy and magnetic field behave similarly in that they are compatible with a $k^{+1 / 3}$ power law in the inertial range. As the dynamo saturates, the magnetic energy in the larger scales increases and the spectrum is subsequently compatible with a $k^{-1}$ slope. Thus, there is a similarity between the spectra of enstrophy and magnetic field during the kinematic regime. Yet once the dynamo has saturated these spectra are different.

Recent simulations of turbulent dynamos in the presence of differential rotation (Brandenburg et al. 1995a) have shown that shear can lead to strong toroidal magnetic fields that are large scale and which reverse direction on a long timescale in a cyclic manner. There the turbulence is driven by a magnetic shear flow instability, which we believe to be important in accretion discs. In future work we plan to combine shear and convection, hoping that this will provide a useful model for the solar dynamo. 
We thank Peter Fox, Dmitry Sokoloff and Alain Vincent for useful comments on the manuscript. The computations were carried out on the Cray-XMP/432 of the Centre for Scientific Computing, Espoo, Finland, the Cray-3 at NCAR in Boulder, Colorado, the Cray-2 at CCVR (Centre de Calcul Vectoriel pour la Recherche), Palaiseau, France, and the Cray C92 at UNI $\bullet$ C in Copenhagen, Denmark. This work was supported in part by the Advanced Study Program at NCAR (A.B.), SERC (A.B. and R.L.J.), the Danish National Research Foundation through its establishment of the Theoretical Astrophysics Center ( $\AA$.N.), NASA grant NAGW1695 (R.F.S.), and the EC Human Capital and Mobility (Networks) project "Late type stars: activity, magnetism, turbulence" No. ERBCHRXCT940483 (A.B., M.R. and I.T.).

\section{REFERENCES}

ANDRÉ, J.-C. \& LESIEUR, M. 1977 Influence of helicity on high Reynolds number isotropic turbulence. $J$. Fluid Mech. 81, 187-207.

BATCHELOR, G. K. 1950 On the spontaneous magnetic field in a conducting liquid in turbulent motion. Proc. R. Soc. Lond. A 201, 405-416.

Brandenburg, A., Nordlund, A., Stein, R. F. \& Torkelsson, U. $1995 a$ Dynamo generated turbulence and large scale magnetic fields in a Keplerian shear flow. Astrophys. J. 446, 741754.

Brandenburg, A., Procaccia, I. \& Segel, D. $1995 b$ The size and dynamics of magnetic flux structures in MHD turbulence. Phys. Plasmas 2, 1148-1156.

Castaing, B., Gunaratne, G., Heslot, F., Kadanofy, L., Libchaber, A., Thomae, S., Wu, X.-Z., ZALESKI, S. \& ZANETTI, G. 1989 Scaling of hard thermal turbulence in Rayleigh-Bénard convection. J. Fluid Mech. 204, 1-30.

Cattaneo, F., Hughes, D. W. \& Weiss, N. O. 1991 What is a stellar dynamo? Mon. Not. R. Astron. Soc. 253, 479-484.

Dittrich, P. K., Molchanov, S. A., Ruzmaikin, A. A. \& Sokoloff, D. D. 1988 Stationary distribution of the value of the magnetic field in a random flow. Magnetohydrodynamics 24, 274-276.

Doluady, S., Couder, Y. \& Brachet, M. E. 1991 Direct observation of the intermittency of intense vortex filaments in turbulence. Phys. Rev. Lett. 67, 983-986.

Galloway, D. J., Proctor, M. R. E. \& Weiss, N. O. 1977 Formation of intense magnetic fields near the surface of the Sun. Nature 266, 686-689.

GilmaN, P. A. 1983 Dynamically consistent nonlinear dynamos driven by convection in a rotating spherical shell. II. Dynamos with cycles and strong feedbacks. Astrophys. J. Suppl. 53, 243-268.

Glatzmaier, G. A. 1985 Numerical simulations of stellar convective dynamos. II. Field propagation in the convection zone. Astrophys. J. 291, 300-307.

Hurlburt, N. E., ToOmre, J. \& Massaguer, J. M. 1984 Two-dimensional compressible convection extending over multiple scale heights. Astrophys. J. 282, 557-573.

Hurlburt, N. E., ToOmre, J. \& Massaguer, J. M. 1986 Nonlinear compressible convection penetrating into stable layers and producing internal gravity waves. Astrophys. J. 311, 563577.

Hyman, J. M. 1979 A method of lines approach to the numerical solution of conservation laws. In Advances in Computation Methods for Partial Differential Equations, Vol. III (ed. R. Vichnevetsky \& R. S. Stepleman), pp. 313-343. Publ. IMACS.

Jennings, R. L., Brandenburg, A., Nordlund, A. \& Stein, R. F. 1992 Evolution of a magnetic flux tube in two dimensional penetrative convection. Mon. Not. R. Astron. Soc. 259, 465-473.

KERR, R. M. 1985 Higher-order derivative correlations and the alignment of small-scale structures in isotropic numerical turbulence. J. Fluid Mech. 153, 31-58.

KERR, R. M. 1987 Histograms of helicity and strain in numerical turbulence. Phys. Rev. Lett. 59, $783-786$.

Kida, S., Yanase, S. \& Mizushima, J. 1991 Statistical properties of MHD turbulence and turbulent dynamo. Phys. Fluids A 3, 457-465. 
Kraichnan, R. H. 1965 Inertial-range spectrum of hydromagnetic turbulence. Phys. Fluids 8 , $1385-1387$.

Kraichnan, R. H. 1990 Models of intermittency in hydrodynamic turbulence. Phys. Rev. Lett. 65, 575-578.

Lesieur, M. 1990 Turbulence in Fluids, 2nd Edn. Martinius Nijhoff.

MeneguzzI, M. \& Pouquet, A. 1989 Turbulent dynamos driven by convection. J. Fluid Mech. 205, 297-312.

MofFAT, H. K. 1961 The amplification of a weak magnetic applied magnetic field by turbulence in fluids of moderate conductivity. $J$. Fluid Mech. 11, 625-635.

Nordlund, A., Brandenburg, A., Jennings, R. L., Rieutord, M., Ruokolainen, J., Stein, R. F. \& TuOMINEN, I. 1992 Dynamo action in stratified convection with overshoot. Astrophys. $J$. 392, 647-652 (referred to herein as Paper I).

Nordlund, A. \& Stein, R. F. 1990 3-D Simulations of solar and stellar convection and magnetoconvection. Comput. Phys. Commun. 59, 119-125.

Parker, E. N. 1979 The instability of a horizontal magnetic field in an atmosphere stable against convection. Astrophys. Space Sci. 62, 135-142.

PARKER, E. N. 1984 Magnetic buoyancy and the escape of magnetic fields from stars. Astrophys. J. 281, 839-845.

Pelz, R. B., Yakhot, V., Orszag, S. A., Shtilman, L. \& Levich, E. 1985 Velocity-vorticity patterns in turbulent flow. Phys. Rev. Lett. 54, 2505-2508.

Petrovay, K. 1991 Topological pumping in the lower overshoot layer The Sun and Cool Stars: Activity, Magnetism, Dynamos (ed. I. Tuominen, D. Moss \& G. Rüdiger). Lecture Notes in Physics, vol. 380 , pp. 67-70. Springer.

Pouquet A., Frisch, U. \& LÉORAT, J. 1976 Strong MHD helical turbulence and the nonlinear dynamo effect. $J$. Fluid Mech. 77, 321-354.

Pouquet A. \& Patterson, G. S. 1978 Numerical simulation of helical magnetohydrodynamic turbulence. J. Fluid Mech. 85, 305-323.

Pumir, A., Shraiman, B. \& Siggia, E. D. 1991 Exponential tails and random advection. Phys. Rev. Lett. 66, 2984-2987.

RIEUTORD, M. \& ZAHN, J.-P. 1995 Turbulent plumes in stellar convective envelopes. Astron. Astrophys. 296, 127-138.

Ruzmaikin, A. A. \& ShukuRov, A. M. 1982 Spectrum of the galactic magnetic field. Astrophys. Space Sci. 82, 397-407.

Schüssler, M. 1977 On buoyant magnetic flux tubes in the solar convection zone. Astron. Astrophys. 56, 439-442.

SCHÜSSLER, M. 1984 On the structure of magnetic fields in the solar convection zone. In The Hydromagnetics of the Sun (ed. T. D. Guyenne \& J. J. Hunt), pp. 67-75. ESA SP-220.

SHE, Z,-S., JACKSON, E. \& ORSZAG, S. A. 1990 Intermittent vortex structures in homogeneous isotropic turbulence. Nature 344, 226-228.

ToOmRe, J., Zahn, J.-P., Latour, J. \& SPIEgel, E. A. 1976 Stellar convection theory. II. Single-mode study of the second convection zone in an A-type star. Astrophys. J. 207, 545-563.

Vainshtein, S. I. \& Zeldovich, YA. B. 1972 Origin of magnetic fields in astrophysics. Sov. Phys. Usp. 15, 159-172.

Valdettaro, L. \& Meneguzzi, M. 1991 Turbulent dynamos driven by convection inside spherical shells The Sun and Cool Stars: Activity. Magnetism, Dynamos (ed. I. Tuominen, D. Moss \& G. Rïdiger). Lecture Notes in Physics, vol. 380, pp. 80-85. Springer.

VINCENT, A. \& MfNEGUZZI, M. 1991 The spatial structure and statistical properties of homogeneous turbulence. J. Fluid Mech. 225, 1-20.

Vincent, A. \& MENEGUZZI, M. 1994 On the dynamics of vortex tubes in homogeneous turbulence. J. Fluid Mech. 258, 245-254.

Zeldovich, Ya. B., Molchanov, S. A., Ruzmaikin, A. A. \& Sokoloff, D. D. 1987 Intermittency in random media. Sov. Phys. Usp. 30, 353-369.

Zeldovich, YA. B., Ruzmaikin, A. A. \& Sokoloff, D. D. 1990 The Almighty Chance. World Scientific. 